ESAIM: COCV 20 (2014) 362-388

DOI: $10.1051 / \mathrm{cocv} / 2013067$
ESAIM: Control, Optimisation and Calculus of Variations

www.esaim-cocv.org

\title{
GLOBAL MINIMIZER OF THE GROUND STATE FOR TWO PHASE CONDUCTORS IN LOW CONTRAST REGIME*,**
}

\author{
Antoine LAURAin ${ }^{1}$
}

\begin{abstract}
The problem of distributing two conducting materials with a prescribed volume ratio in a ball so as to minimize the first eigenvalue of an elliptic operator with Dirichlet conditions is considered in two and three dimensions. The gap $\varepsilon$ between the two conductivities is assumed to be small (low contrast regime). The main result of the paper is to show, using asymptotic expansions with respect to $\varepsilon$ and to small geometric perturbations of the optimal shape, that the global minimum of the first eigenvalue in low contrast regime is either a centered ball or the union of a centered ball and of a centered ring touching the boundary, depending on the prescribed volume ratio between the two materials.
\end{abstract}

Mathematics Subject Classification. 49Q10, 35P15, 49R05, 47A55, 34E10.

Received April 2, 2013. Revised June 24, 2013.

Published online March 3, 2014.

\section{INTRODUCTION}

Shape optimization for eigenvalues of elliptic operators provides interesting and challenging mathematical problems; see [9] and the references therein for an overview. In this paper we are considering the problem of minimizing the first eigenvalue of an elliptic operator with respect to the distribution of two conducting materials in a fixed domain. Let $\Omega \subset \mathbb{R}^{d}$ be an open bounded set. Let $m$ be a given positive number, $0<m<|\Omega|$, where $|\Omega|$ is the Lebesgue measure of $\Omega$. Two materials with conductivities $\alpha$ and $\beta(0<\alpha<\beta)$ are distributed in arbitrary disjoint measurable subsets $A$ and $B$, respectively, of $\Omega$ so that $A \cup B=\Omega$ and $|B|=m$. Consider the two-phases eigenvalue problem:

$$
\begin{aligned}
-\operatorname{div}(\sigma \nabla u) & =\lambda u \text { in } \Omega, \\
u & =0 \text { on } \partial \Omega,
\end{aligned}
$$

Keywords and phrases. Shape optimization, eigenvalue optimization, two-phase conductors, low contrast regime, asymptotic analysis.

* Financial support by the DFG Research Center Matheon "Mathematics for key technologies" through the MATHEON-Project C37 "Shape/Topology optimization methods for inverse problems".

** The author would like to thank Rajesh Mahadevan for the invitation to stay at the University de Concepción in September 2012 and the fruitful discussions on this problem.

1 Technische Universität Berlin, Sekretariat MA 4-5, Straße des 17. Juni 136, 10623 Berlin, Germany.

laurain@math.tu-berlin.de 
with the conductivity $\sigma:=\alpha \chi_{A}+\beta \chi_{B}$, where $\chi_{A}$ is the indicator function of $A$. Let $\lambda$ be the first eigenvalue of (1.1)-(1.2) and $u$ the associated eigenvector. The Rayleigh quotient for $\lambda$ is

$$
\lambda=\min _{u \in H_{0}^{1}(\Omega)} \frac{\int_{\Omega} \sigma|\nabla u|^{2}}{\int_{\Omega} u^{2}}=\min _{u \in H_{0}^{1}(\Omega),\|u\|_{2}=1} \int_{\Omega} \sigma|\nabla u|^{2},
$$

where $\|u\|_{2}$ denotes the $L^{2}$-norm of $u$. In this paper we consider the case $\Omega=\mathbb{B}(0,1)$, the case $\Omega=\mathbb{B}(0, R)$ being readily deduced from our results, and we are interested in the dependence of $\lambda$ on $A$ and $B$. Since $A=\Omega \backslash B, \lambda$ may be described as a function of $B$ and we occasionally write $\lambda=\lambda(B)$. We consider the problem of minimizing $\lambda(B)$ with the constraint that the two phases are to be distributed in fixed proportions:

$$
\begin{aligned}
\operatorname{minimize} & \lambda(B) \\
\text { subject to } & B \in \mathcal{B}
\end{aligned}
$$

where

$$
\mathcal{B}:=\{B \subset \Omega, B \text { measurable, }|B|=m\} .
$$

The existence of a solution to the problem (1.4)-(1.6) remains an open question for a general $\Omega$. Minimizing sequences may develop microstructural patterns and the original problem may have to be relaxed to include microstructural designs. Existence of a solution and optimality conditions in the class of relaxed designs has been discussed by Cox and Lipton in [6]. However, the original problem (1.4)-(1.6) may still have a solution for particular geometries as is the case when $\Omega$ is a ball. When $\Omega=\mathbb{B}(0, R)$ is a ball, the existence of a radially symmetric optimal set has been proved in [1], using rearrangement techniques and a comparison result for Hamilton-Jacobi equations and later, only using rearrangement techniques in [4]. Even in this case an explicit solution to the problem was not known. It was conjectured in $[4,5]$, in dimension greater than one, that the solution $B^{*}$ to this problem is a ball $\mathrm{B}\left(0, r^{*}\right)$ as in the one-dimensional case; see [10]. Recent numerical tests [7] and theoretical results came up recently to reinforce the conjecture. It was shown in [5], using second order shape derivative calculus, that such a configuration is a local minimum for the problem when the volume constraint $m$ is small enough.

However, in [3] it has been proved that the conjecture is not true in general. Indeed, the optimal domain $B^{*}$ cannot be a ball when $\alpha$ and $\beta$ are close to each other and $m$ is sufficiently large. This negative result is provided by an asymptotic expansion of the eigenvalue with respect to $\beta-\alpha$ as $\beta \rightarrow \alpha$, the so-called "low contrast regime" also assumed in this paper, which allows to approximate (1.4)-(1.6) by a simpler optimization problem. However the question of minimizers of $\lambda(B)$ even in the low contrast regime was still left open.

In this paper we prove first, when $\Omega=\mathbb{B}(0,1)$, the convergence as $\beta \rightarrow \alpha$ in the sense of characteristic functions of the minimizing sets either to a centered ball $B^{*}=\mathbb{B}\left(0, r^{*}\right)$ when $m$ is below a certain threshold $\bar{m}$ or to $B^{*}=\mathbb{B}\left(0, \xi^{0}\right) \cup \mathbb{B}(0,1) \backslash \overline{\mathbb{B}\left(0, \xi^{1}\right)}$, i.e. the union of a centered ball and a centered ring touching the boundary of $\Omega$, when $m>\bar{m}$. Then the main result of the paper is to show that $B^{*}$ or $B_{\varepsilon}^{*}=\mathbb{B}\left(0, \xi_{\varepsilon}^{0}\right) \cup \mathbb{B}(0,1) \backslash \overline{\mathbb{B}\left(0, \xi_{\varepsilon}^{1}\right)}$ are actually global minimizers of $\lambda(B)$ when $\beta-\alpha$ is small enough. The main ideas to obtain these results are first to exploit the additional regularity provided by the radial symmetry, which yields a stronger convergence of the eigenfunction as $\beta \rightarrow \alpha$, which in turn provides the set convergence. To prove that $B^{*}$ or $B_{\varepsilon}^{*}$ is a global minimizer, we compare its eigenvalue with the eigenvalue of all possible radially symmetric sets in a small neighbourhood of $B^{*}$ or $B_{\varepsilon}^{*}$ using an asymptotic expansion with respect to small geometric perturbations of sets.

In Section 2 the low contrast regime is described and some known results are recalled. In Section 3 the set convergence of the solutions to (1.4) $-(1.5)$ as $\varepsilon:=\beta-\alpha \rightarrow 0$ is proved, using the additional regularity thanks to the radial symmetry. In Section 4 and 5 we prove that there exists $\varepsilon_{0}$ such that the ball $B^{*}$ is a solution of (1.4) -(1.5) when $m<\bar{m}$ and such that $B_{\varepsilon}^{*}$ is a solution of (1.4)-(1.5) when $m>\bar{m}$. 


\section{LOW CONTRAST REGIME}

In this section we recall some known results required for our analysis. Throughout the paper we assume the so-called low contrast regime, i.e. the conductivities of the two materials, $\alpha$ and $\beta$, are close to each other: $\beta=\beta_{\varepsilon}:=\alpha+\varepsilon$ with $\varepsilon>0$ small. If the material with conductivity $\beta_{\varepsilon}$ occupies the sub-domain $B$ of $\Omega$, the conductivity coefficient is, in this case,

$$
\sigma=\sigma_{\varepsilon}(B):=\alpha \chi_{A}+\beta_{\varepsilon} \chi_{B}=\alpha+\varepsilon \chi_{B} .
$$

Let $\lambda_{\varepsilon}(B)$ be the first eigenvalue in the problem

$$
\begin{aligned}
-\operatorname{div}\left(\sigma_{\varepsilon}(B) \nabla u_{\varepsilon}\right) & =\lambda_{\varepsilon}(B) u_{\varepsilon} \text { in } \Omega, \\
u_{\varepsilon} & =0 \text { on } \partial \Omega
\end{aligned}
$$

for the conductivity $\sigma_{\varepsilon}(B)$. It is well-known, from the Kreln-Rutman Theorem [11], that the first eigenvalue of a linear elliptic operator is simple and the corresponding eigenfunction is of constant sign (and is the only eigenvalue whose eigenfunction does not change sign). Thus one may choose the eigenfunction $u_{\varepsilon}=u_{\varepsilon}(B)$ corresponding to $\lambda_{\varepsilon}(B)$ to be positive and normalize it using the condition

$$
\int_{\Omega}\left(u_{\varepsilon}\right)^{2}=1
$$

In this way, $u_{\varepsilon}$ is uniquely defined. For fixed $B$, we claim that both $\lambda_{\varepsilon}(B)$ and $u_{\varepsilon}(B)$ analytically depend on the parameter $\varepsilon$. This result is classical in the perturbation theory of eigenvalues and follows readily, for instance, from Theorem 3, Chapter 2.5 of Rellich [12]. This justifies the ansätze

$$
\begin{aligned}
& \lambda_{\varepsilon}(B)=\lambda_{0}(B)+\varepsilon \lambda_{1}(B)+\ldots \\
& u_{\varepsilon}(B)=u_{0}(B)+\varepsilon u_{1}(B)+\ldots
\end{aligned}
$$

In fact, $\lambda_{0}(B)=\lambda_{0}$ is the first eigenvalue of the problem

$$
\begin{aligned}
-\alpha \Delta u_{0} & =\lambda u_{0} \quad \text { in } \Omega, \\
u_{0} & =0 \text { on } \partial \Omega .
\end{aligned}
$$

The function $u_{0}$ is the positive eigenfunction corresponding to $\lambda_{0}$ and satisfies the normalization condition $\int_{\Omega} u_{0}^{2}=1$. Therefore $\lambda_{0}(B)=\lambda_{0}$ and $u_{0}(B)=u_{0}$ are independent of $B$. For the next term we have ([3], Prop. 2.2):

Proposition 2.1. In ansatz $(2.5), \lambda_{1}(B)$ is given explicitly in terms of $u_{0}$ as follows

$$
\lambda_{1}(B)=\int_{B}\left|\nabla u_{0}\right|^{2} \mathrm{~d} x
$$

The convergence of the series in (2.6) holds in the space $H_{0}^{1}(\Omega)$. The following result can be found in ([3], Thm. 2.3):

Theorem 2.2. For $\varepsilon>0$ sufficiently small, there exists a constant $c$ independent of $\varepsilon$ and $B$ such that

$$
\left\|u_{\varepsilon}(B)-u_{0}\right\|_{H_{0}^{1}(\Omega)} \leq c \varepsilon^{\frac{1}{2}} \quad \forall B \in \mathcal{B} .
$$

The results above are valid for any open set $\Omega$. In this paper we are interested in the particular case $\Omega=\mathbb{B}(0,1)$ and we need the following result which can be found in [4]:

Theorem 2.3. Let $\Omega=\mathbb{B}(0,1)$. The problem (1.4)-(1.5) admits a radially symmetric solution.

Applying Theorem 2.3, we denote $B_{\varepsilon}^{*}$ a radially symmetric solution of (1.4)-(1.5) for $\beta=\beta_{\varepsilon}$. 

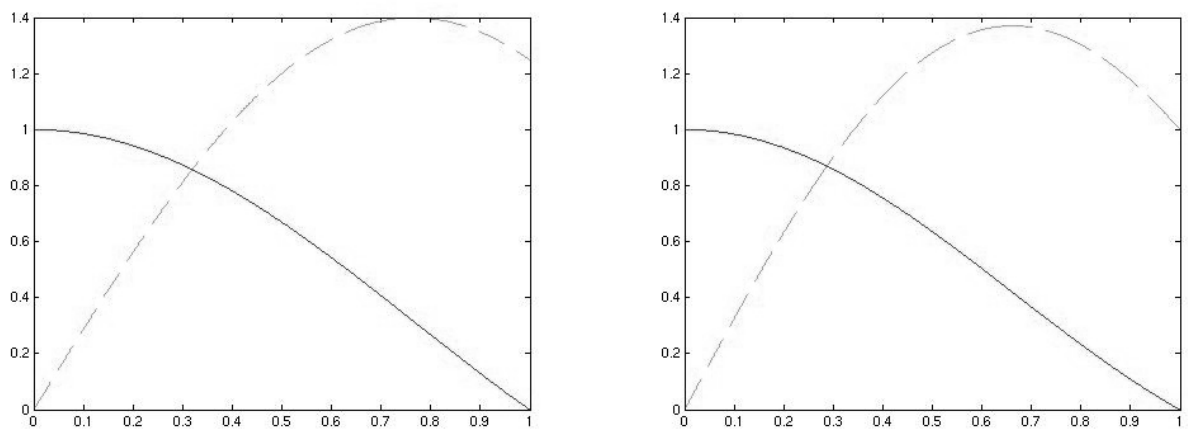

Figure 1. Functions $\bar{u}_{0}(r)$ (plain), and $w_{1}(r)=-\bar{u}_{0}^{\prime}(r)$ (dashed) in dimensions $d=2$ (left) and $d=3$ (right). The constant $r_{d}^{1}$ is such that $w_{1}$ is increasing on $\left[0, r_{d}^{1}\right]$ and decreasing on $\left[r_{d}^{1}, 1\right]$, and $r_{d}^{0}$ is such that $w_{1}\left(r_{d}^{0}\right)=w_{1}(1)$.

\section{Convergence of Minimizing Sets}

In this section and the rest of the paper we assume $\Omega=\mathbb{B}(0,1) \subset \mathbb{R}^{d}, d=2,3$ to be the ball of center 0 and radius 1 . The results can be straightforwardly extended to the case $\Omega=\mathbb{B}(0, R)$. In this case, the solution $u_{0}$ of $(2.7)-(2.8)$ is radial and smooth. In view of Theorem 2.3 we assume $B$ is radially symmetric. By setting $\bar{u}_{0}(r):=u_{0}(x)$ where $r:=|x|$, equation $(2.7)-(2.8)$ becomes, using the Laplacian in polar $(r, \theta)$ or spherical $(r, \theta, \varphi)$ coordinates, for $d=2,3$,

$$
\begin{aligned}
r^{2} \bar{u}_{0}^{\prime \prime}(r)+(d-1) r \bar{u}_{0}^{\prime}(r)+r^{2} \frac{\lambda_{0}}{\alpha} \bar{u}_{0}(r) & =0, \\
\bar{u}_{0}^{\prime}(0)=0, \bar{u}_{0}(1) & =0 .
\end{aligned}
$$

where the boundary conditions (3.2) correspond to the continuity of the gradient at the origin and the Dirichlet condition on the boundary, respectively. The solution of this equation is

$$
\begin{array}{ll}
\bar{u}_{0}(r)=J_{0}\left(\eta_{d} r\right) & \text { if } d=2, \\
\bar{u}_{0}(r)=j_{0}\left(\eta_{d} r\right) & \text { if } d=3,
\end{array}
$$

where $J_{0}$ and $j_{0}$ denote Bessel functions of the first and second kind, respectively and $\eta_{d}(d=2,3)$ are their respective zeros; see [13] for details on Bessel functions. The behaviour of $\bar{u}_{0}$ is depicted in Figure 1. Let $\omega_{d}$ denote the volume of the unit ball, i.e. we have $\omega_{d}=\pi$ for $d=2$ and $\omega_{d}=4 \pi / 3$ for $d=3$. Let $r_{d}^{0}$ and $r_{d}^{1}$ be the constants defined in the caption of Figure 1 . Not that these constants can be easily approximated with an arbitrary precision thanks to the explicit representation of Bessel functions. Introduce the radius $r_{d}^{*}:=\left(m / \omega_{d}\right)^{1 / d}$ and the threshold $\bar{m}_{d}:=\omega_{d}\left(r_{d}^{0}\right)^{d}$.

Proposition 3.1. When $\Omega=\mathbb{B}(0,1) \subset \mathbb{R}^{d}, d=2,3$, the unique rotationally symmetric optimal domain $B^{*}$ which minimizes $\lambda_{1}(B)$ over $B \in \mathcal{B}$ is of two possible types:

- Type I: If $m \leq \bar{m}_{d}$ then $B^{*}=\mathbb{B}\left(0, r_{d}^{*}\right)$ or,

- Type II: If $m>\bar{m}_{d}$ then there exists $\xi^{0}$ and $\xi^{1}$ with

$$
r_{d}^{*}<\xi^{0}<\xi^{1}<1
$$

and $B^{*}=\mathbb{B}\left(0, \xi^{0}\right) \cup\left(\mathbb{B}(0,1) \backslash \overline{\mathrm{B}\left(0, \xi^{1}\right)}\right)$. 
In this section we show that the global minimizer $B_{\varepsilon}^{*}$ of $\lambda_{\varepsilon}(B)$ converges in the sense of characteristic functions to $B^{*}$ given in Proposition 3.1. For simplicity we will write $r^{*}=r_{d}^{*}$ and $\bar{m}=\bar{m}_{d}$ from now on. We need the following inequality; see [8], Theorem 330 and [2] for details. In all of our subsequent computations, $c$ denotes a generic constant independent of $\varepsilon$.

Theorem 3.2 (Generalized Hardy's inequality). If $p>1, q \neq 1, f(x) \geq 0$, and $F(x)$ is defined as

$$
F(x)=\left\{\begin{array}{l}
\int_{0}^{x} f(t) \mathrm{d} t \quad \text { for } q>1, \\
\int_{x}^{\infty} f(t) \mathrm{d} t \quad \text { for } q<1
\end{array}\right.
$$

then

$$
\int_{0}^{\infty} x^{-q} F(x)^{p} \mathrm{~d} x<\left(\frac{p}{|q-1|}\right)^{p} \int_{0}^{\infty} x^{-q}(x f(x))^{p} \mathrm{~d} x
$$

unless $f \equiv 0$.

We start by proving some regularity results for the solution $u_{\varepsilon}$.

Theorem 3.3. Assume $\Omega=\mathbb{B}(0,1)$ and $B$ is radially symmetric. The functions $u_{\varepsilon}$ and $u_{0}$ are in $W^{1, \infty}(\Omega)$ and there exists $\varepsilon_{0}>0$ such that for all $\varepsilon<\varepsilon_{0}$ we have

$$
\left\|\sigma_{\varepsilon} \nabla u_{\varepsilon}-\sigma_{0} \nabla u_{0}\right\|_{L^{\infty}(\Omega)} \leq c \sqrt{\varepsilon}
$$

and

$$
\left\|\nabla u_{\varepsilon}-\nabla u_{0}\right\|_{L^{\infty}(\Omega)} \leq c \sqrt{\varepsilon} .
$$

Proof. The proof is divided into two steps. In the first step we prove that $u_{\varepsilon} \in L^{\infty}(\Omega)$ and in the second step we prove the $L^{\infty}$-convergence for the gradients.

Step 1. In view of Theorem 2.2 we have $u_{\varepsilon} \rightarrow u_{0}$ in $H_{0}^{1}(\Omega)$. The key feature of the proof is to introduce the function $\bar{u}_{\varepsilon}(r):=u_{\varepsilon}(x)$ where $r=|x|$ in polar or spherical coordinates and to show that $\bar{u}_{\varepsilon}^{\prime} \rightarrow \bar{u}_{0}^{\prime}$ in $L^{\infty}([0,1])$. According to ([3], Thm. 2.3) we have $\left\|u_{\varepsilon}\right\|_{H^{1}(\Omega)} \leq c$ where $c$ is independent from $\varepsilon$. The system (1.1)-(1.2) leads to the equations for $\bar{u}_{\varepsilon}$ and $r \in(0,1)$ :

$$
\begin{aligned}
-r^{-(d-1)} \frac{\mathrm{d}}{\mathrm{d} r}\left[r^{d-1} \sigma_{\varepsilon}(r) \bar{u}_{\varepsilon}^{\prime}(r)\right] & =\lambda_{\varepsilon} \bar{u}_{\varepsilon}(r), \\
\bar{u}_{\varepsilon}(1) & =0 .
\end{aligned}
$$

Set $g_{\varepsilon}:=\sigma_{\varepsilon} \bar{u}_{\varepsilon}^{\prime}-\sigma_{0} \bar{u}_{0}^{\prime}$. Taking the difference between (3.5) and (3.5) at $\varepsilon=0$ and multiplying with $-r^{d-1}$ we get

$$
\frac{\mathrm{d}}{\mathrm{d} r}\left[r^{d-1} g_{\varepsilon}(r)\right]=-r^{d-1}\left(\lambda_{\varepsilon} \bar{u}_{\varepsilon}(r)-\lambda_{0} \bar{u}_{0}(r)\right)
$$

Integrating $(3.7)$ on $(0, r)$ we get

$$
r^{d-1} g_{\varepsilon}(r)-\lim _{t \rightarrow 0} t^{d-1} g_{\varepsilon}(t)=-\int_{0}^{r} t^{d-1}\left(\lambda_{\varepsilon} \bar{u}_{\varepsilon}(t)-\lambda_{0} \bar{u}_{0}(t)\right) \mathrm{d} t .
$$

Since $\left\|u_{\varepsilon}\right\|_{H^{1}(\Omega)} \leq c$ we have $\left\|r^{(d-1) / 2} \bar{u}_{\varepsilon}^{\prime}\right\|_{L^{2}(0,1)} \leq c$ using polar or spherical coordinates. This implies that $r^{d-1} \bar{u}_{\varepsilon}^{\prime}(r) \rightarrow 0$ as $r \rightarrow 0$ and in turn

$$
\lim _{t \rightarrow 0} t^{d-1} g_{\varepsilon}(t)=0 .
$$


Dividing by $r^{d-1}$,taking the $L^{2}$-norm and using the Cauchy-Schwarz inequality we get

$$
\begin{aligned}
\left\|g_{\varepsilon}\right\|_{L^{2}(0,1)}^{2} & =\left\|r^{1-d} \int_{0}^{r} t^{d-1}\left(\lambda_{\varepsilon} \bar{u}_{\varepsilon}(t)-\lambda_{0} \bar{u}_{0}(t)\right) \mathrm{d} t\right\|_{L^{2}(0,1)}^{2} \\
& \leq \int_{r=0}^{1} r^{-2(d-1)}\left\|t^{d-1}\left(\lambda_{\varepsilon} \bar{u}_{\varepsilon}-\lambda_{0} \bar{u}_{0}\right)\right\|_{L^{1}(0, r)}^{2} \mathrm{~d} r \\
& \leq\left\|\lambda_{\varepsilon} \bar{u}_{\varepsilon}-\lambda_{0} \bar{u}_{0}\right\|_{L^{2}(0,1)}^{2} \int_{0}^{1} r^{-2(d-1)}\left\|t^{d-1}\right\|_{L^{2}(0, r)}^{2} \mathrm{~d} r \\
& \leq(2 d-2)^{-1}\left\|\lambda_{\varepsilon} \bar{u}_{\varepsilon}-\lambda_{0} \bar{u}_{0}\right\|_{L^{2}(0,1)}^{2} .
\end{aligned}
$$

According to Theorem 2.2 we have $\left\|u_{\varepsilon}-u_{0}\right\|_{H^{1}(\Omega)} \leq c \sqrt{\varepsilon}$ for $\varepsilon$ small enough. Passing to polar or spherical coordinates this implies

$$
\left\|r^{(d-1) / 2}\left(\bar{u}_{\varepsilon}-\bar{u}_{0}\right)\right\|_{L^{2}(0,1)} \leq c \sqrt{\varepsilon} \text { and }\left\|r^{(d-1) / 2}\left(\bar{u}_{\varepsilon}^{\prime}-\bar{u}_{0}^{\prime}\right)\right\|_{L^{2}(0,1)} \leq c \sqrt{\varepsilon} .
$$

Using Lemma 3.2 with $q=0, p=2$ and $f=\bar{u}_{\varepsilon}^{\prime}-\bar{u}_{0}^{\prime}$ we obtain

$$
\int_{0}^{1}\left(\bar{u}_{\varepsilon}-\bar{u}_{0}\right)^{2} \mathrm{~d} r<4 \int_{0}^{1} r^{2}\left(\bar{u}_{\varepsilon}^{\prime}-\bar{u}_{0}^{\prime}\right)^{2} \mathrm{~d} r
$$

Therefore we have

$$
\left\|\bar{u}_{\varepsilon}-\bar{u}_{0}\right\|_{L^{2}(0,1)} \leq c\left\|r^{(d-1) / 2}\left(\bar{u}_{\varepsilon}^{\prime}-\bar{u}_{0}^{\prime}\right)\right\|_{L^{2}(0,1)} \leq c \sqrt{\varepsilon},
$$

and in a similar way

$$
\left\|\bar{u}_{\varepsilon}\right\|_{L^{2}(0,1)} \leq c .
$$

Since $\left|\lambda_{\varepsilon}-\lambda_{0}\right| \leq c \varepsilon$ as $\varepsilon \rightarrow 0$ we get

$$
\left\|g_{\varepsilon}\right\|_{L^{2}(0,1)} \leq c \sqrt{\varepsilon} \text { as } \varepsilon \rightarrow 0 .
$$

Performing a similar calculation for $\sigma_{\varepsilon} \bar{u}_{\varepsilon}^{\prime}$ and using $\sigma_{\varepsilon} \geq \alpha$ we get

$$
\left\|\bar{u}_{\varepsilon}^{\prime}\right\|_{L^{2}(0,1)}^{2} \leq \alpha^{-2}\left\|\sigma_{\varepsilon} \bar{u}_{\varepsilon}^{\prime}\right\|_{L^{2}(0,1)}^{2} \leq \alpha^{-2}(2 d-2)^{-1}\left\|\lambda_{\varepsilon} \bar{u}_{\varepsilon}\right\|_{L^{2}(0,1)}^{2} \leq c
$$

where $c$ is a constant independent on $\varepsilon$. Using a Sobolev imbedding we also get

$$
\left\|\bar{u}_{\varepsilon}\right\|_{L^{\infty}(0,1)} \leq c\left\|\bar{u}_{\varepsilon}\right\|_{H^{1}(0,1)} \leq c\left\|\bar{u}_{\varepsilon}^{\prime}\right\|_{L^{2}(0,1)} \leq c .
$$

Step 2. Integrating and taking the $L^{\infty}$-norm we get

$$
\begin{aligned}
\left\|g_{\varepsilon}\right\|_{L^{\infty}(0,1)} & =\left\|r^{1-d} \int_{0}^{r} t^{d-1}\left(\lambda_{\varepsilon} \bar{u}_{\varepsilon}(t)-\lambda_{0} \bar{u}_{0}(t)\right) \mathrm{d} t\right\|_{L^{\infty}(0,1)} \\
& \leq\left\|\lambda_{\varepsilon} \bar{u}_{\varepsilon}-\lambda_{0} \bar{u}_{0}\right\|_{L^{\infty}(0,1)}\left\|r^{1-d} \int_{0}^{r} t^{d-1} \mathrm{~d} t\right\|_{L^{\infty}(0,1)} \\
& \leq c\left|\lambda_{\varepsilon}-\lambda_{0}\right|\left\|\bar{u}_{\varepsilon}\right\|_{L^{\infty}(0,1)}+\lambda_{0}\left\|\bar{u}_{\varepsilon}-\bar{u}_{0}\right\|_{L^{\infty}(0,1)} .
\end{aligned}
$$

Performing a similar estimate for $\sigma_{\varepsilon} \bar{u}_{\varepsilon}^{\prime}$ we obtain

$$
\left\|\sigma_{\varepsilon} \bar{u}_{\varepsilon}^{\prime}\right\|_{L^{\infty}(0,1)} \leq\left|\lambda_{\varepsilon}\right|\left\|\bar{u}_{\varepsilon}\right\|_{L^{\infty}(0,1)} .
$$

Now we show that $\left\|\bar{u}_{\varepsilon}-\bar{u}_{0}\right\|_{L^{\infty}(0,1)} \rightarrow 0$. Using (3.13) gives

$$
\left\|\bar{u}_{\varepsilon}^{\prime}\right\|_{L^{\infty}(0,1)} \leq c .
$$


Next, writing

$$
\begin{aligned}
\left\|\bar{u}_{\varepsilon}^{\prime}-\bar{u}_{0}^{\prime}\right\|_{L^{2}(0,1)} & =\frac{1}{\sigma_{0}}\left\|\sigma_{0} \bar{u}_{\varepsilon}^{\prime}-\sigma_{0} \bar{u}_{0}^{\prime}\right\|_{L^{2}(0,1)} \\
& \leq \frac{1}{\sigma_{0}}\left\|g_{\varepsilon}\right\|_{L^{2}(0,1)}+\frac{\varepsilon}{\sigma_{0}}\left\|\chi_{B} \bar{u}_{\varepsilon}^{\prime}\right\|_{L^{2}(0,1)},
\end{aligned}
$$

and using that $\left\|\bar{u}_{\varepsilon}^{\prime}\right\|_{L^{2}(0,1)}$ is uniformly bounded with respect to $\varepsilon$ we get

$$
\left\|\bar{u}_{\varepsilon}^{\prime}-\bar{u}_{0}^{\prime}\right\|_{L^{2}(0,1)} \leq c \sqrt{\varepsilon} \text { as } \varepsilon \rightarrow 0 .
$$

Since $\bar{u}_{\varepsilon}(1)=\bar{u}_{0}(1)=0$, we may apply Poincaré's inequality and we get

$$
\left\|\bar{u}_{\varepsilon}-\bar{u}_{0}\right\|_{H^{1}(0,1)} \leq c \sqrt{\varepsilon} \text { as } \varepsilon \rightarrow 0 .
$$

Due to Sobolev imbeddings in one dimension we have

$$
\left\|\bar{u}_{\varepsilon}-\bar{u}_{0}\right\|_{L^{\infty}(0,1)} \leq c\left\|\bar{u}_{\varepsilon}-\bar{u}_{0}\right\|_{H^{1}(0,1)} \leq c \sqrt{\varepsilon} \text { as } \varepsilon \rightarrow 0 .
$$

Finally, using the estimate for $g_{\varepsilon}$ and (3.11) we obtain

$$
\left\|g_{\varepsilon}\right\|_{L^{\infty}(0,1)} \leq c \sqrt{\varepsilon} \text { as } \varepsilon \rightarrow 0 .
$$

Next, writing

$$
\begin{aligned}
\left\|\bar{u}_{\varepsilon}^{\prime}-\bar{u}_{0}^{\prime}\right\|_{L^{\infty}(0,1)} & =\frac{1}{\sigma_{0}}\left\|\sigma_{0} \bar{u}_{\varepsilon}^{\prime}-\sigma_{0} \bar{u}_{0}^{\prime}\right\|_{L^{\infty}(0,1)} \\
& \leq \frac{1}{\sigma_{0}}\left\|g_{\varepsilon}\right\|_{L^{\infty}(0,1)}+\frac{\varepsilon}{\sigma_{0}}\left\|\chi_{B} \bar{u}_{\varepsilon}^{\prime}\right\|_{L^{\infty}(0,1)} .
\end{aligned}
$$

Finally using (3.14) and (3.15) we get $\left\|\bar{u}_{\varepsilon}^{\prime}-\bar{u}_{0}^{\prime}\right\|_{L^{\infty}(0,1)} \leq c \sqrt{\varepsilon}$ as $\varepsilon \rightarrow 0$. Going back to the function $u_{\varepsilon}(x)=$ $\bar{u}_{\varepsilon}(|x|)$ we have obtained indeed

$$
\left\|\nabla u_{\varepsilon}-\nabla u_{0}\right\|_{L^{\infty}(\Omega)} \leq c \sqrt{\varepsilon} .
$$

The fact that $u_{\varepsilon}$ and $u_{0}$ are in $W^{1, \infty}(\Omega)$ derives directly from (3.11) and (3.14).

Remark 3.4. Since we have shown that $\left\|\bar{u}_{\varepsilon}\right\|_{H^{1}(0,1)}$ is uniformly bounded with respect to $\varepsilon$, using classical Sobolev imbeddings we also have the Hölder regularity $\bar{u}_{\varepsilon} \in \mathcal{C}^{0,1 / 2}([0,1])$.

Thanks to Theorem 3.3 we can now prove the set convergence of the minimizer $B_{\varepsilon}^{*}$ of $\lambda_{\varepsilon}(B)$ to $B^{*}$. First of all we prove that the set $B_{\varepsilon}^{*}$ is close to $B^{*}$ in an appropriate sense. We need some preliminary results. Introduce the quantity

$$
\mathfrak{M}(B, c)=\left|\left\{x:\left|\nabla u_{B}(x)\right| \leq c\right\}\right|
$$

where $u_{B}$ is the solution of $(1.1)-(1.2)$. The proof of the two following results can be found in ([3], Lem. 3.1) and ([3], Prop. 2.7):

Lemma 3.5. The function $\mathfrak{M}(B, c)$ is non-decreasing with respect to $c$ and is such that $\mathfrak{M}(B, c) \rightarrow 0$ as $c \rightarrow 0$ and $\mathfrak{M}(B, c) \rightarrow|\Omega|$ as $c \rightarrow \infty$. Furthermore, it is a right-continuous function. It is also left continuous at any $c$ if and only if the Lebesgue measure of $\left\{x:\left|\nabla u_{B}(x)\right|=c\right\}$ is zero.

Proposition 3.6. For $\Omega=\mathbb{B}(0,1)$, the level set $\left\{\left|\nabla u_{0}\right|=c\right\}$ has zero measure for each $c \geq 0$.

We start with optimal sets of Type I, i.e. when $m<\bar{m}$. 
Theorem 3.7. Let $B \subset \Omega$ be an arbitrary radially symmetric measurable set and $m<\bar{m}$. For all $\delta>0$, there exists $\varepsilon_{0}=\varepsilon_{0}(\delta)>0$ and $B_{\delta}^{*}$ radially symmetric and containing the origin such that $\left|B_{\delta}^{*}\right|=m$,

$$
\mathbb{B}\left(0, r^{*}-\delta\right) \subset B_{\delta}^{*} \subset \mathbb{B}\left(0, r^{*}+\delta\right)
$$

and for all $0<\varepsilon \leq \varepsilon_{0}(\delta)$ we have the inequality

$$
\lambda_{\varepsilon}\left(B_{\delta}^{*}\right) \leq \lambda_{\varepsilon}(B)
$$

Proof. Let $\eta>0$ be chosen, in view of Theorem 3.3, there exists $\varepsilon_{0}(\eta)>0$ such that

$$
\left|\nabla u_{\varepsilon}(x)-\nabla u_{0}(x)\right|<\eta \text { for all } x \in \Omega \text { and } \varepsilon<\varepsilon_{0}(\eta) .
$$

Denote

$$
\begin{aligned}
\mathfrak{B}_{\varepsilon}^{c} & :=\left\{x \in \Omega,\left|\nabla u_{\varepsilon}(x)\right|<c\right\}, \\
B_{\varepsilon}^{c} & :=\left\{x \in \Omega,\left|\nabla u_{\varepsilon}(x)\right| \leq c\right\}, \\
B_{0}^{c} & :=\left\{x \in \Omega,\left|\nabla u_{0}(x)\right| \leq c\right\} .
\end{aligned}
$$

Using (3.19) we have the following inclusions

$$
\begin{aligned}
B_{0}^{c} & \subset\left\{x \in \Omega,\left|\nabla u_{0}(x)\right|<c+\eta-\left|\nabla u_{\varepsilon}(x)-\nabla u_{0}(x)\right|\right\} \\
& =\left\{x \in \Omega,\left|\nabla u_{0}(x)\right|+\left|\nabla u_{\varepsilon}(x)-\nabla u_{0}(x)\right|<c+\eta\right\} \\
& \subset\left\{x \in \Omega,\left|\nabla u_{\varepsilon}(x)\right|<c+\eta\right\}=\mathfrak{B}_{\varepsilon}^{c+\eta} \subset B_{\varepsilon}^{c+\eta} .
\end{aligned}
$$

In a similar way we may write

$$
\begin{aligned}
\mathfrak{B}_{\varepsilon}^{c-\eta} \subset B_{\varepsilon}^{c-\eta} & \subset\left\{x \in \Omega,\left|\nabla u_{\varepsilon}(x)\right|+\left|\nabla u_{0}(x)-\nabla u_{\varepsilon}(x)\right| \leq c-\eta+\eta\right\} \\
& \subset\left\{x \in \Omega,\left|\nabla u_{0}(x)\right| \leq c\right\}=B_{0}^{c} .
\end{aligned}
$$

Thus we have obtained

$$
\mathfrak{B}_{\varepsilon}^{c-\eta} \subset B_{\varepsilon}^{c-\eta} \subset B_{0}^{c} \subset \mathfrak{B}_{\varepsilon}^{c+\eta} \subset B_{\varepsilon}^{c+\eta}
$$

Lemma 3.5 and Proposition 3.6 imply that $\left|B_{0}^{c}\right|$ is continuous, consequently, using the assumption $m<\bar{m}$ and Proposition 3.1, there exists a $c^{*}$ such that $B_{0}^{c^{*}}=\mathbb{B}\left(0, r^{*}\right)$ and $\left|B_{0}^{c^{*}}\right|=m$. Taking $c=c^{*}$ in (3.20) we get

$$
\left|\mathfrak{B}_{\varepsilon}^{c^{*}-\eta}\right| \leq\left|B_{\varepsilon}^{c^{*}-\eta}\right| \leq m \leq\left|\mathfrak{B}_{\varepsilon}^{c^{*}+\eta}\right| \leq\left|B_{\varepsilon}^{c^{*}+\eta}\right| .
$$

Since $\left|B_{\varepsilon}^{c}\right|$ and $\left|\mathfrak{B}_{\varepsilon}^{c}\right|$ are increasing (but not necessarily continuous) functions there exists $c_{\varepsilon}$ such that

$$
c^{*}-\eta \leq c_{\varepsilon} \leq c^{*}+\eta,
$$

and

$$
\left|\mathfrak{B}_{\varepsilon}^{c_{\varepsilon}}\right| \leq m \text { and }\left|B_{\varepsilon}^{c_{\varepsilon}}\right| \geq m,
$$

We apply (3.20) first with $c=c_{\varepsilon}+\eta$ and then $c=c_{\varepsilon}-\eta$ and we obtain the inclusions

$$
B_{0}^{c_{\varepsilon}-\eta} \subset \mathfrak{B}_{\varepsilon}^{c_{\varepsilon}} \subset B_{\varepsilon}^{c_{\varepsilon}} \subset B_{0}^{c_{\varepsilon}+\eta} .
$$

Finally applying (3.22) we get

$$
B_{0}^{c^{*}-2 \eta} \subset \mathfrak{B}_{\varepsilon}^{c_{\varepsilon}} \subset B_{\varepsilon}^{c_{\varepsilon}} \subset B_{0}^{c^{*}+2 \eta} .
$$


Now let $\delta>0$ be given. Assumption $m<\bar{m}$ and Proposition 3.1 (see Fig. 1) ensure that there exists $\eta=\eta(\delta)>0$ such that

$$
\begin{aligned}
\mathbb{B}\left(0, r^{*}-\delta\right) & \subset B_{0}^{c^{*}-2 \eta}, \\
B_{0}^{c^{*}+2 \eta} & \subset \mathbb{B}\left(0, r^{*}+\delta\right) .
\end{aligned}
$$

Note that the strict inequality $m<\bar{m}$ is necessary otherwise the above property may not be true for all $\delta$. In view of (3.23) and (3.24), there exists a radially symmetric set $B_{\delta}^{*}$ such that $\left|B_{\delta}^{*}\right|=m$ and

$$
\mathbb{B}\left(0, r^{*}-\delta\right) \subset B_{0}^{c^{*}-2 \eta} \subset \mathfrak{B}_{\varepsilon}^{c_{\varepsilon}} \subset B_{\delta}^{*} \subset B_{\varepsilon}^{c_{\varepsilon}} \subset B_{0}^{c^{*}+2 \eta} \subset \mathbb{B}\left(0, r^{*}+\delta\right) .
$$

which yields (3.17).

To prove (3.18), consider the decompositions

$$
\begin{aligned}
B & =\left(B \cap B_{\delta}^{*}\right) \cup\left(B \cap\left(B_{\delta}^{*}\right)^{c}\right), \\
B_{\delta}^{*} & =\left(B \cap B_{\delta}^{*}\right) \cup\left(B^{c} \cap B_{\delta}^{*}\right) .
\end{aligned}
$$

Since $|B|=\left|B_{\delta}^{*}\right|=m$ we have with the above decompositions

$$
\left|B \cap\left(B_{\delta}^{*}\right)^{c}\right|=\left|B^{c} \cap B_{\delta}^{*}\right| .
$$

Observing that $\left|\nabla u_{\varepsilon}\right| \geq c_{\varepsilon}$ on $\left(B_{\delta}^{*}\right)^{c}$ and $\left|\nabla u_{\varepsilon}\right| \leq c_{\varepsilon}$ on $B_{\delta}^{*}$, we may write

$$
\begin{aligned}
\int_{B}\left|\nabla u_{\varepsilon}\right|^{2} & =\int_{B \cap B_{\delta}^{*}}\left|\nabla u_{\varepsilon}\right|^{2}+\int_{B \cap\left(B_{\delta}^{*}\right)^{c}}\left|\nabla u_{\varepsilon}\right|^{2} \\
& \geq \int_{B \cap B_{\delta}^{*}}\left|\nabla u_{\varepsilon}\right|^{2}+c_{\varepsilon}^{2}\left|B \cap\left(B_{\delta}^{*}\right)^{c}\right| \\
& =\int_{B \cap B_{\delta}^{*}}\left|\nabla u_{\varepsilon}\right|^{2}+c_{\varepsilon}^{2}\left|B^{c} \cap B_{\delta}^{*}\right| \\
& \geq \int_{B \cap B_{\delta}^{*}}\left|\nabla u_{\varepsilon}\right|^{2}+\int_{B^{c} \cap B_{\delta}^{*}}\left|\nabla u_{\varepsilon}\right|^{2}=\int_{B_{\delta}^{*}}\left|\nabla u_{\varepsilon}\right|^{2} .
\end{aligned}
$$

As a consequence

$$
\begin{aligned}
\lambda_{\varepsilon}(B) & =\alpha \int_{\Omega}\left|\nabla u_{\varepsilon}\right|^{2}+(\beta-\alpha) \int_{B}\left|\nabla u_{\varepsilon}\right|^{2} \\
& \geq \alpha \int_{\Omega}\left|\nabla u_{\varepsilon}\right|^{2}+(\beta-\alpha) \int_{B_{\delta}^{*}}\left|\nabla u_{\varepsilon}\right|^{2} \\
& \geq \min _{u \in H_{0}^{1}(\Omega),\|u\|_{2}=1}\left(\alpha \int_{\Omega}|\nabla u|^{2}+(\beta-\alpha) \int_{B_{\delta}^{*}}|\nabla u|^{2}\right)=\lambda_{\varepsilon}\left(B_{\delta}^{*}\right),
\end{aligned}
$$

and the inequality (3.18) is proved.

Remark 3.8. Note that $B_{\delta}^{*}$ may be barely measurable, i.e. may have a fractal structure, even though it is radially symmetric. 
We also have the following result for optimal sets of Type II:

Theorem 3.9. Let $B \subset \Omega$ an arbitrary measurable set and $m>\bar{m}$. For all $\delta>0$, there exists $\varepsilon_{0}=\varepsilon_{0}(\delta)$ and $B_{\delta}^{*}$ radially symmetric and containing the origin such that $\left|B_{\delta}^{*}\right|=m, B_{\delta}^{*}=B_{\delta, 0}^{*} \cup B_{\delta, 1}^{*}$,

$$
\begin{aligned}
\mathrm{B}\left(0, \xi^{0}-\delta\right) & \subset B_{\delta, 0}^{*} \subset \mathbb{B}\left(0, \xi^{0}+\delta\right), \\
\mathbb{B}(0,1) \backslash \overline{\mathrm{B}\left(0, \xi^{1}+\delta\right)} & \subset B_{\delta, 1}^{*} \subset \mathbb{B}(0,1) \backslash \overline{\mathrm{B}\left(0, \xi^{1}-\delta\right),}
\end{aligned}
$$

and for all $0<\varepsilon \leq \varepsilon_{0}(\delta)$ we have the inequality

$$
\lambda_{\varepsilon}\left(B_{\delta}^{*}\right) \leq \lambda_{\varepsilon}(B)
$$

where $\left(\xi_{0}, \xi_{1}\right)$ are given in Proposition 3.1.

Proof. The proof is left to the reader as it is almost identical to the proof of Theorem 3.9 except that it is based on the Type II optimizers appearing in Proposition 3.1.

Corollary 3.10. If $m<\bar{m}$ and $\varepsilon_{k} \rightarrow 0$ as $k \rightarrow \infty$, then there exists a sequence of solutions $B_{\varepsilon_{k}}$ of (1.4)-(1.5) such that

$$
\chi_{B_{\varepsilon_{k}}} \rightarrow \chi_{\mathbb{B}\left(0, r^{*}\right)} \text { in } L^{p}(\Omega), \quad p \in[1, \infty[,
$$

i.e. $B_{\varepsilon_{k}}$ converges to $\mathbb{B}\left(0, r^{*}\right)$ in the sense of characteristic functions.

If $m>\bar{m}$ and $\varepsilon_{k} \rightarrow 0$ as $k \rightarrow \infty$, then there exists a sequence of solutions $B_{\varepsilon_{k}}$ of (1.4)-(1.5) such that

$$
\chi_{B_{\varepsilon_{k}}} \rightarrow \chi_{B^{*}} \text { in } L^{p}(\Omega), \quad p \in[1, \infty[
$$

where $B^{*}=\mathbb{B}\left(0, \xi^{0}\right) \cup\left(\mathbb{B}(0,1) \backslash \overline{\mathbb{B}\left(0, \xi^{1}\right)}\right)$ as defined in Proposition 3.1.

Proof. In a first step assume $m<\bar{m}$. We define the function

$$
\varepsilon(\delta):=\delta \min _{\nu \geq \delta} \varepsilon_{0}(\nu)
$$

where $\varepsilon_{0}(\delta)$ is given by Theorem 3.7. Clearly, $\min _{\nu \geq \delta} \varepsilon_{0}(\nu)$ is a nondecreasing function and consequently $\varepsilon$ is a strictly increasing function of $\delta$ since $\min _{\nu \geq \delta} \varepsilon_{0}(\nu)>0$ for $\delta>0$ and we also have $\varepsilon(\delta) \rightarrow 0$ as $\delta \rightarrow 0$. Therefore there exists a strictly increasing inverse function $\hat{\delta}(\varepsilon)$ such that $\varepsilon(\hat{\delta}(\varepsilon))=\varepsilon$ and $\hat{\delta}(\varepsilon) \rightarrow 0$ as $\varepsilon \rightarrow 0$.

According to Theorem 3.7, for a given $\varepsilon$ there exists $B_{\hat{\delta}(\varepsilon)}^{*}$ radially symmetric and containing the origin such that $\left|B_{\hat{\delta}(\varepsilon)}^{*}\right|=m$,

$$
\mathbb{B}\left(0, r^{*}-\hat{\delta}(\varepsilon)\right) \subset B_{\hat{\delta}(\varepsilon)}^{*} \subset \mathbb{B}\left(0, r^{*}+\hat{\delta}(\varepsilon)\right)
$$

and we have the inequality

$$
\lambda_{\varepsilon}\left(B_{\hat{\delta}(\varepsilon)}^{*}\right) \leq \lambda_{\varepsilon}(B)
$$

Let $B_{\varepsilon}$ be a radially symmetric set solution of (1.4)-(1.5) for a given $\varepsilon$. We know that such a solution exists according to [4]. Taking $B=B_{\varepsilon}$ in (3.33) we get

$$
\lambda_{\varepsilon}\left(B_{\hat{\delta}(\varepsilon)}^{*}\right) \leq \lambda_{\varepsilon}\left(B_{\varepsilon}\right)
$$

therefore $B_{\hat{\delta}(\varepsilon)}^{*}$ is also a solution of (1.4)-(1.5). In view of (3.32) and $\hat{\delta}(\varepsilon) \rightarrow 0$ as $\varepsilon \rightarrow 0$ we clearly obtain

$$
\chi_{B_{\hat{\delta}(\varepsilon)}^{*}} \rightarrow \chi_{\mathbb{B}\left(0, r^{*}\right)} \text { in } L^{p}(\Omega), \quad p \in[1, \infty[,
$$

and the claim follows. 
In a similar way, if we assume $m>\bar{m}$ we may apply Theorem 3.9 to obtain

$$
\chi_{B_{\hat{\delta}(\varepsilon)}^{*}} \rightarrow \chi_{B^{*}} \text { in } L^{p}(\Omega), \quad p \in[1, \infty[,
$$

where $B^{*}=\mathbb{B}\left(0, \xi^{0}\right) \cup\left(\mathbb{B}(0,1) \backslash \overline{\mathrm{B}\left(0, \xi^{1}\right)}\right)$.

We can actually prove that $B_{\varepsilon_{k}}=\mathbb{B}\left(0, r^{*}\right)$ or $\mathbb{B}\left(0, \xi^{0}\right) \cup\left(\mathbb{B}(0,1) \backslash \overline{\mathbb{B}\left(0, \xi^{1}\right)}\right)$ when $\varepsilon_{k}$ is small enough. This is the purpose of the next sections.

\section{INEQUALITy FOR TyPe I OPTIMUM}

In this section we assume $m<\bar{m}$ so that we are in the framework provided by Theorem 3.7.

\subsection{Description of the method}

The main result of this section is to prove the existence of a threshold $\varepsilon_{0}>0$ such that

$$
\lambda_{\varepsilon}\left(B^{*}\right) \leq \lambda_{\varepsilon}(B),
$$

for all $B \in \mathcal{B}$ and $\varepsilon \leq \varepsilon_{0}$, where $B^{*}=\mathbb{B}\left(0, r^{*}\right)$ as given by Proposition 3.1. This implies that $B^{*}$ is a solution of (1.4)-(1.5) in low contrast regime, i.e. for $\varepsilon \leq \varepsilon_{0}$. In view of the proof of Corollary 3.10, for all $\varepsilon>0$ there exists a $\delta(\varepsilon)>0$ such that

$$
\lambda_{\varepsilon}\left(B_{\delta(\varepsilon)}^{*}\right) \leq \lambda_{\varepsilon}(B)
$$

holds with $\delta(\varepsilon) \rightarrow 0$ as $\varepsilon \rightarrow 0$ and $\delta(\varepsilon)$ strictly increasing. Therefore to conclude we require the other inequality

$$
\lambda_{\varepsilon}\left(B^{*}\right) \leq \lambda_{\varepsilon}\left(B_{\delta(\varepsilon)}^{*}\right) .
$$

The difficulty in obtaining this last inequality is that we do not have any information on $B_{\delta(\varepsilon)}^{*}$ apart from the fact that it is "close" to $B^{*}$ in the sense of Theorem 3.7. Fortunately it is just enough to perform an asymptotic expansion of the eigenvalue with respect to $\delta(\varepsilon)$. In this section we show that there exists $\varepsilon_{0}>0$ and $\delta_{0}>0$ such that for all $0<\varepsilon \leq \varepsilon_{0}$ and $0<\delta \leq \delta_{0}$ we have

$$
\lambda_{\varepsilon}\left(B^{*}\right) \leq \lambda_{\varepsilon}\left(B_{\delta}\right)
$$

where $B_{\delta}$ is any radially symmetric set satisfying

$$
\mathbb{B}\left(0, r^{*}-\delta\right) \subset B_{\delta} \subset \mathbb{B}\left(0, r^{*}+\delta\right) .
$$

In particular one can choose $B_{\delta}=B_{\delta(\varepsilon)}^{*}$ for $\varepsilon$ small enough, and combining (4.2)-(4.4) we get

$$
\lambda_{\varepsilon}\left(B^{*}\right) \leq \lambda_{\varepsilon}\left(B_{\delta}\right)=\lambda_{\varepsilon}\left(B_{\delta(\varepsilon)}^{*}\right) \leq \lambda_{\varepsilon}(B),
$$

which gives (4.1). To prove (4.4) we aim at obtaining an asymptotic expansion of $\lambda_{\varepsilon}\left(B_{\delta}\right)$ with respect to $(\varepsilon, \delta)$ of the type

$$
\lambda_{\varepsilon}\left(B_{\delta}\right)=\lambda_{\varepsilon}\left(B^{*}\right)+\rho(\delta) \bar{\lambda}_{\varepsilon}+\mathcal{R}(\varepsilon, \delta),
$$

with $\rho(\delta)>0, \rho(\delta) \rightarrow 0$ and $\mathcal{R}(\varepsilon, \delta) / \rho(\delta) \rightarrow 0$ uniformly as $(\delta) \rightarrow 0$. Proving then that $\bar{\lambda}_{\varepsilon} \geq 0$ we get (4.4) for $(\varepsilon, \delta)$ small enough.

We introduce $A_{\delta}:=\Omega \backslash B_{\delta}$ and $A^{*}:=\Omega \backslash B^{*}$. We clearly have

$$
\chi_{B_{\delta}}-\chi_{B^{*}}=-\left(\chi_{A_{\delta}}-\chi_{A^{*}}\right)
$$


and

$$
\int_{\Omega} \chi_{B_{\delta}}-\chi_{B^{*}}=m-m=0
$$

We introduce the sets $I_{\delta}^{ \pm}$such that

$$
\begin{aligned}
& I_{\delta}^{+}:=\left\{x \in \Omega \mid \chi_{B_{\delta}}(x)-\chi_{B^{*}}(x)=1\right\} \\
& I_{\delta}^{-}:=\left\{x \in \Omega \mid \chi_{B_{\delta}}(x)-\chi_{B^{*}}(x)=-1\right\} .
\end{aligned}
$$

It is clear that

$$
\chi_{B_{\delta}}-\chi_{B^{*}}=\chi_{I_{\delta}^{+}}-\chi_{I_{\delta}^{-}},
$$

and due to assumption (4.5) we have

$$
\begin{aligned}
& I_{\delta}^{+} \subset \mathbb{B}\left(0, r^{*}+\delta\right) \backslash \mathbb{B}\left(0, r^{*}\right), \\
& I_{\delta}^{-} \subset \mathbb{B}\left(0, r^{*}\right) \backslash \mathbb{B}\left(0, r^{*}-\delta\right) .
\end{aligned}
$$

\subsection{Asymptotic expansion}

Denote $\left(u_{\varepsilon, \delta}, \lambda_{\varepsilon, \delta}\right)$ the eigenpair solution of

$$
\begin{aligned}
-\operatorname{div}\left(\sigma_{\varepsilon, \delta} \nabla u_{\varepsilon, \delta}\right) & =\lambda_{\varepsilon, \delta} u_{\varepsilon, \delta} \text { in } \Omega, \\
u_{\varepsilon, \delta} & =0 \text { on } \partial \Omega,
\end{aligned}
$$

with $\sigma_{\varepsilon, \delta}=\alpha \chi_{A_{\delta}}+\beta_{\varepsilon} \chi_{B_{\delta}}$. The main difficulty in writing the asymptotic analysis of $u_{\varepsilon, \delta}$ with respect to $(\varepsilon, \delta)$ is the lack of regularity of $u_{\varepsilon, \delta}$, since $\sigma_{\varepsilon, \delta}$ is only piecewise constant and $B_{\delta}$ is possibly barely measurable. To overcome this difficulty, we advantageously use the fact that $\sigma_{\varepsilon, \delta} \nabla u_{\varepsilon, \delta}$ has more regularity than $\nabla u_{\varepsilon, \delta}$ in view of (4.14). Therefore an important strategy in the proofs of the asymptotic expansion is to often replace $\nabla u_{\varepsilon, \delta}$ by $\sigma_{\varepsilon, \delta}^{-1}\left(\sigma_{\varepsilon, \delta} \nabla u_{\varepsilon, \delta}\right)$ in the computations in order to write Taylor expansions for $\sigma_{\varepsilon, \delta} \nabla u_{\varepsilon, \delta}$ or related quantities.

In asymptotic analysis, one typically starts with an ansatz with respect to the asymptotically small parameters. In our case we would like to obtain an expansion with respect to the small parameter $\delta$ for fixed $\varepsilon$ of the type:

$$
\begin{aligned}
& u_{\varepsilon, \delta}=u_{\varepsilon, 0}+\rho(\delta) u_{\varepsilon, 1}+o(\rho(\delta)), \\
& \lambda_{\varepsilon, \delta}=\lambda_{\varepsilon, 0}+\rho(\delta) \lambda_{\varepsilon, 1}+o(\rho(\delta)),
\end{aligned}
$$

In our case it is unclear what should $\rho(\delta)$ be even if $\rho(\delta)$ will be determined further in (4.36), so it is preferable in a first step to not separate the $\delta$-dependent and $\varepsilon$-dependent term in ansätze (4.18)-(4.19). Thus we rather decompose $u_{\varepsilon, \delta}$ and $\lambda_{\varepsilon, \delta}$ in the following way:

$$
\begin{aligned}
& u_{\varepsilon, \delta}=u_{\varepsilon, 0}+w_{\varepsilon, \delta}+\mathcal{R}_{u}(\varepsilon, \delta), \\
& \lambda_{\varepsilon, \delta}=\lambda_{\varepsilon, 0}+\mu_{\varepsilon, \delta}+\mathcal{R}_{\lambda}(\varepsilon, \delta),
\end{aligned}
$$

where $u_{\varepsilon, 0}, w_{\varepsilon, \delta}$ and $\mathcal{R}_{u}(\varepsilon, \delta)$ satisfy

$$
\begin{aligned}
-\operatorname{div}\left(\sigma_{\varepsilon, 0} \nabla u_{\varepsilon, 0}\right) & =\lambda_{\varepsilon, 0} u_{\varepsilon, 0} \text { in } \Omega, \\
u_{\varepsilon, 0} & =0 \text { on } \partial \Omega, \\
-\operatorname{div}\left(\sigma_{\varepsilon, 0} \nabla w_{\varepsilon, \delta}\right)-\lambda_{\varepsilon, 0} w_{\varepsilon, \delta}= & \operatorname{div}\left(\frac{\left(\sigma_{\varepsilon, \delta}-\sigma_{\varepsilon, 0}\right) \sigma_{\varepsilon, 0}}{\sigma_{\varepsilon, \delta}} \nabla u_{\varepsilon, 0}\right) \\
& +\mu_{\varepsilon, \delta} u_{\varepsilon, 0} \text { in } \Omega, \\
w_{\varepsilon, \delta}= & 0 \text { on } \partial \Omega .
\end{aligned}
$$




$$
\begin{aligned}
-\operatorname{div}\left(\sigma_{\varepsilon, \delta} \nabla \mathcal{R}_{u}\right)-\lambda_{\varepsilon, \delta} \mathcal{R}_{u}= & \operatorname{div}\left(\left(\sigma_{\varepsilon, \delta}-\sigma_{\varepsilon, 0}\right)\left[\frac{\left(\sigma_{\varepsilon, \delta}-\sigma_{\varepsilon, 0}\right)}{\sigma_{\varepsilon, \delta}} \nabla u_{\varepsilon, 0}+\nabla w_{\varepsilon, \delta}\right]\right) \\
& +\mu_{\varepsilon, \delta} w_{\varepsilon, \delta}+\mathcal{R}_{\lambda} u_{\varepsilon, \delta} \text { in } \Omega \\
w_{\varepsilon, \delta}= & 0 \text { on } \partial \Omega .
\end{aligned}
$$

The decomposition is chosen so that $\mathcal{R}_{u}=\mathcal{O}\left(w_{\varepsilon, \delta}\right)$ and $\mathcal{R}_{\lambda}=\mathcal{O}\left(\mu_{\varepsilon, \delta}\right)$ as $(\varepsilon, \delta) \rightarrow 0$ in a sense that will be determined in Lemma 4.2 and Theorem 4.5. Note that these problems are well-defined in the weak sense, with the right-hand side in $H^{-1}(\Omega)$ and that the solutions $\sigma_{\varepsilon, 0}, w_{\varepsilon, \delta}, \mathcal{R}_{u}$ are in $H^{1}(\Omega)$.

According to the Fredholm alternative, (4.22)-(4.23) has a solution only if the right-hand side of (4.22) is orthogonal to $u_{\varepsilon, 0}$ which yields

$$
\int_{\Omega} \operatorname{div}\left(\frac{\left(\sigma_{\varepsilon, \delta}-\sigma_{\varepsilon, 0}\right) \sigma_{\varepsilon, 0}}{\sigma_{\varepsilon, \delta}} \nabla u_{\varepsilon, 0}\right) u_{\varepsilon, 0} \mathrm{~d} x+\mu_{\varepsilon, \delta} \int_{\Omega} u_{\varepsilon, 0}^{2} \mathrm{~d} x=0
$$

This gives using the normalization condition and Green's formula

$$
\mu_{\varepsilon, \delta}=\int_{\Omega} \frac{\left(\sigma_{\varepsilon, \delta}-\sigma_{\varepsilon, 0}\right) \sigma_{\varepsilon, 0}}{\sigma_{\varepsilon, \delta}}\left|\nabla u_{\varepsilon, 0}\right|^{2} \mathrm{~d} x
$$

In a similar way $(4.24)-(4.25)$ has a solution only if the right-hand side of (4.24) is orthogonal to $u_{\varepsilon, \delta}$ which yields

$$
\int_{\Omega} \operatorname{div}\left(\left(\sigma_{\varepsilon, \delta}-\sigma_{\varepsilon, 0}\right)\left[\frac{\left(\sigma_{\varepsilon, \delta}-\sigma_{\varepsilon, 0}\right)}{\sigma_{\varepsilon, \delta}} \nabla u_{\varepsilon, 0}+\nabla w_{\varepsilon, \delta}\right]\right) u_{\varepsilon, \delta}+\mu_{\varepsilon, \delta} \int_{\Omega} w_{\varepsilon, \delta} u_{\varepsilon, \delta}+\mathcal{R}_{\lambda} \int_{\Omega} u_{\varepsilon, \delta}^{2}=0 .
$$

Using the normalization condition for $u_{\varepsilon, \delta}$ and an integration by parts we get

$$
\mathcal{R}_{\lambda}=\mathcal{R}_{\lambda}^{1}+\mathcal{R}_{\lambda}^{2}+\mathcal{R}_{\lambda}^{3}
$$

where

$$
\begin{aligned}
& \mathcal{R}_{\lambda}^{1}:=-\mu_{\varepsilon, \delta} \int_{\Omega} w_{\varepsilon, \delta} u_{\varepsilon, \delta}, \quad \mathcal{R}_{\lambda}^{2}:=\int_{\Omega} \frac{\left(\sigma_{\varepsilon, \delta}-\sigma_{\varepsilon, 0}\right)^{2}}{\sigma_{\varepsilon, \delta}} \nabla u_{\varepsilon, 0} \cdot \nabla u_{\varepsilon, \delta}, \\
& \mathcal{R}_{\lambda}^{3}:=\int_{\Omega}\left(\sigma_{\varepsilon, \delta}-\sigma_{\varepsilon, 0}\right) \nabla w_{\varepsilon, \delta} \cdot \nabla u_{\varepsilon, \delta} .
\end{aligned}
$$

Using (4.7) we get

$$
\begin{aligned}
\sigma_{\varepsilon, \delta}-\sigma_{\varepsilon, 0} & =\alpha\left(\chi_{A_{\delta}}-\chi_{A^{*}}\right)+\beta_{\varepsilon}\left(\chi_{B_{\delta}}-\chi_{B^{*}}\right) \\
& =\left(\beta_{\varepsilon}-\alpha\right)\left(\chi_{B_{\delta}}-\chi_{B^{*}}\right)=\varepsilon\left(\chi_{B_{\delta}}-\chi_{B^{*}}\right) .
\end{aligned}
$$

Note that so far the expansion is valid for any $B_{\delta} \subset \Omega$ (not necessarily symmetric) and any $\Omega \subset \mathbb{R}^{d}$. The next step consists in providing the expansion of $\mu_{\varepsilon, \delta}$ with respect to $(\varepsilon, \delta)$ and to obtain uniform estimates for the remainders $\mathcal{R}_{u}$ and $\mathcal{R}_{\lambda}$ as $\chi_{B_{\delta}} \rightarrow \chi_{B^{*}}$ when $\Omega$ is a disk and $B_{\delta}$ is radially symmetric.

\section{3. $L^{\infty}$-estimates}

From now on we assume $\Omega=\mathbb{B}(0,1), B_{\delta}$ is radially symmetric and satisfies assumption (4.5). In this section we prove $L^{\infty}$-estimates for $w_{\varepsilon, \delta}$ and $\mathcal{R}_{u}$ that will be required later to estimate $\mathcal{R}_{\lambda}$. For simplicity, in what follows $c$ denotes a generic constant independent of $(\varepsilon, \delta)$. 
Lemma 4.1. The function $w_{\varepsilon, \delta}$ is in $\mathcal{C}^{0}(\bar{\Omega})$ and we have the estimate

$$
\left\|w_{\varepsilon, \delta}\right\|_{L^{\infty}(\Omega)} \leq c\left(\varepsilon\left|I_{\delta}^{+}\right|^{1 / 2}+\left|\mu_{\varepsilon, \delta}\right|\right), .
$$

where $c>0$ is a constant independent of $(\varepsilon, \delta)$.

Proof. In view of (4.20)-(4.21) we have $u_{\varepsilon, 0} \in H_{0}^{1}(\Omega)$ and consequently the right-hand side of (4.22)-(4.23) is in $H^{-1}(\Omega)$ since $\sigma_{\varepsilon, \delta}$ is bounded from below. Therefore $w_{\varepsilon, \delta} \in H_{0}^{1}(\Omega)$ and we actually have the standard estimate for elliptic partial differential equations:

$$
\left\|w_{\varepsilon, \delta}\right\|_{H^{1}(\Omega)} \leq c\left\|\operatorname{div}\left(\frac{\left(\sigma_{\varepsilon, \delta}-\sigma_{\varepsilon, 0}\right) \sigma_{\varepsilon, 0}}{\sigma_{\varepsilon, \delta}} \nabla u_{\varepsilon, 0}\right)\right\|_{H^{-1}(\Omega)}+c\left|\mu_{\varepsilon, \delta}\right|\left\|u_{\varepsilon, 0}\right\|_{L^{2}(\Omega)}
$$

Using $\left\|u_{\varepsilon, 0}\right\|_{H^{1}(\Omega)} \leq c$ and $\sigma_{\varepsilon, \delta}-\sigma_{\varepsilon, 0}=\varepsilon\left(\chi_{I_{\delta}^{+}}-\chi_{I_{\delta}^{-}}\right)$we obtain

$$
\begin{aligned}
\left\|w_{\varepsilon, \delta}\right\|_{H^{1}(\Omega)} & \leq c\left\|\frac{\left(\sigma_{\varepsilon, \delta}-\sigma_{\varepsilon, 0}\right) \sigma_{\varepsilon, 0}}{\sigma_{\varepsilon, \delta}} \nabla u_{\varepsilon, 0}\right\|_{L^{2}(\Omega)}+c\left|\mu_{\varepsilon, \delta}\right| \\
& \leq c\left(\varepsilon\left|I_{\delta}^{+}\right|^{1 / 2}+\left|\mu_{\varepsilon, \delta}\right|\right) .
\end{aligned}
$$

Passing to polar or spherical coordinates and introducing the notation $\bar{u}_{\varepsilon, 0}(r):=u_{\varepsilon, 0}(x), \bar{w}_{\varepsilon, \delta}(r):=w_{\varepsilon, \delta}(x)$ for the functions depending only on $r$ we obtain

$$
\begin{aligned}
& \left\|r^{(d-1) / 2} \bar{w}_{\varepsilon, \delta}\right\|_{L^{2}(0,1)} \leq c\left(\varepsilon\left|I_{\delta}^{+}\right|^{1 / 2}+\left|\mu_{\varepsilon, \delta}\right|\right) \\
& \left\|r^{(d-1) / 2} \bar{w}_{\varepsilon, \delta}^{\prime}\right\|_{L^{2}(0,1)} \leq c\left(\varepsilon\left|I_{\delta}^{+}\right|^{1 / 2}+\left|\mu_{\varepsilon, \delta}\right|\right)
\end{aligned}
$$

Using Theorem 3.2 and the above inequalities yields

$$
\left\|\bar{w}_{\varepsilon, \delta}\right\|_{L^{2}(0,1)} \leq c\left(\varepsilon\left|I_{\delta}^{+}\right|^{1 / 2}+\left|\mu_{\varepsilon, \delta}\right|\right)
$$

In view of (4.52) we have

$$
\sigma_{\varepsilon, 0} \bar{w}_{\varepsilon, \delta}^{\prime}=-\frac{\left(\sigma_{\varepsilon, \delta}-\sigma_{\varepsilon, 0}\right) \sigma_{\varepsilon, 0}}{\sigma_{\varepsilon, \delta}} \bar{u}_{\varepsilon, 0}^{\prime}+F_{11}(r)
$$

where

$$
F_{11}(r)=-r^{1-d} \int_{0}^{r} t^{d-1}\left(\mu_{\varepsilon, \delta} \bar{u}_{\varepsilon, 0}(t)+\lambda_{\varepsilon, 0} \bar{w}_{\varepsilon, \delta}(t)\right) \mathrm{d} t .
$$

Therefore, in a similar way as in (3.9) we get

$$
\begin{aligned}
\left\|\sigma_{\varepsilon, 0} \bar{w}_{\varepsilon, \delta}^{\prime}\right\|_{L^{2}(0,1)} & \leq\left\|\frac{\left(\sigma_{\varepsilon, \delta}-\sigma_{\varepsilon, 0}\right) \sigma_{\varepsilon, 0}}{\sigma_{\varepsilon, \delta}} \bar{u}_{\varepsilon, 0}^{\prime}\right\|_{L^{2}(0,1)}+c\left\|\mu_{\varepsilon, \delta} \bar{u}_{\varepsilon, 0}+\lambda_{\varepsilon, 0} \bar{w}_{\varepsilon, \delta}\right\|_{L^{2}(0,1)} \\
& \leq c\left(\varepsilon\left|I_{\delta}^{+}\right|^{1 / 2}+\left|\mu_{\varepsilon, \delta}\right|+\left\|\bar{w}_{\varepsilon, \delta}\right\|_{L^{2}(0,1)}\right) .
\end{aligned}
$$

Using (4.29) yields

$$
\alpha\left\|\bar{w}_{\varepsilon, \delta}^{\prime}\right\|_{L^{2}(0,1)} \leq\left\|\sigma_{\varepsilon, 0} \bar{w}_{\varepsilon, \delta}^{\prime}\right\|_{L^{2}(0,1)} \leq c\left(\varepsilon\left|I_{\delta}^{+}\right|^{1 / 2}+\left|\mu_{\varepsilon, \delta}\right|\right)
$$

Combining (4.29) and (4.31) we get

$$
\left\|\bar{w}_{\varepsilon, \delta}\right\|_{H^{1}(0,1)} \leq c\left(\varepsilon\left|I_{\delta}^{+}\right|^{1 / 2}+\left|\mu_{\varepsilon, \delta}\right|\right) .
$$

Using the Sobolev imbedding $H^{1}(0,1) \hookrightarrow L^{\infty}(0,1)$ we obtain

$$
\left\|\bar{w}_{\varepsilon, \delta}\right\|_{L^{\infty}(0,1)} \leq c\left(\varepsilon\left|I_{\delta}^{+}\right|^{1 / 2}+\left|\mu_{\varepsilon, \delta}\right|\right)
$$

and (4.27) follows. From (4.32) we also deduce using a standard Sobolev imbedding that $\bar{w}_{\varepsilon, \delta} \in \mathcal{C}^{0}(0,1)$ and consequently $w_{\varepsilon, \delta} \in \mathcal{C}^{0}(\bar{\Omega})$. 
Lemma 4.2. The function $\mathcal{R}_{u}$ is in $\mathcal{C}^{0}(\bar{\Omega})$ and we have the estimate

$$
\left\|\mathcal{R}_{u}\right\|_{L^{\infty}(\Omega)} \leq c\left(\left(\varepsilon+\left|\mu_{\varepsilon, \delta}\right|\right)\left(\varepsilon\left|I_{\delta}^{+}\right|^{1 / 2}+\left|\mu_{\varepsilon, \delta}\right|\right)+\left|\mathcal{R}_{\lambda}\right|\right),
$$

where $c>0$ is a constant independent of $(\varepsilon, \delta)$.

Proof. In view of Lemma 4.1 and its proof, the right-hand side of $(4.24)-(4.25)$ is in $H^{-1}(\Omega)$ since $\sigma_{\varepsilon, \delta}$ is bounded from below. Therefore $\mathcal{R}_{u} \in H_{0}^{1}(\Omega)$ and we actually have the standard estimate for elliptic equations:

$$
\begin{aligned}
\left\|\mathcal{R}_{u}\right\|_{H^{1}(\Omega)} & \leq c\left\|\operatorname{div}\left(\left(\sigma_{\varepsilon, \delta}-\sigma_{\varepsilon, 0}\right)\left[\frac{\left(\sigma_{\varepsilon, \delta}-\sigma_{\varepsilon, 0}\right)}{\sigma_{\varepsilon, \delta}} \nabla u_{\varepsilon, 0}+\nabla w_{\varepsilon, \delta}\right]\right)\right\|_{H^{-1}(\Omega)} \\
& +c\left\|\mu_{\varepsilon, \delta} w_{\varepsilon, \delta}+\mathcal{R}_{\lambda} u_{\varepsilon, \delta}\right\|_{L^{2}(\Omega)}
\end{aligned}
$$

Using the estimates $\left\|u_{\varepsilon, \delta}\right\|_{L^{2}(\Omega)} \leq c,\left\|u_{\varepsilon, 0}\right\|_{H^{1}(\Omega)} \leq c,(4.28)$ and Lemma 4.1 we get

$$
\begin{aligned}
\left\|\mathcal{R}_{u}\right\|_{H^{1}(\Omega)} & \leq c\left\|\left(\sigma_{\varepsilon, \delta}-\sigma_{\varepsilon, 0}\right)\left[\frac{\left(\sigma_{\varepsilon, \delta}-\sigma_{\varepsilon, 0}\right)}{\sigma_{\varepsilon, \delta}} \nabla u_{\varepsilon, 0}+\nabla w_{\varepsilon, \delta}\right]\right\|_{L^{2}(\Omega)}+c\left|\mu_{\varepsilon, \delta}\right|\left\|w_{\varepsilon, \delta}\right\|_{L^{2}(\Omega)}+\left|\mathcal{R}_{\lambda}\right| \\
& \leq c\left(\varepsilon^{2}\left|I_{\delta}^{+}\right|^{1 / 2}+\varepsilon\left\|w_{\varepsilon, \delta}\right\|_{H^{1}(\Omega)}+\left|\mu_{\varepsilon, \delta}\right|\left\|w_{\varepsilon, \delta}\right\|_{L^{2}(\Omega)}+\left|\mathcal{R}_{\lambda}\right|\right) \\
& \leq c\left(\left(\varepsilon+\left|\mu_{\varepsilon, \delta}\right|\right)\left(\varepsilon\left|I_{\delta}^{+}\right|^{1 / 2}+\left|\mu_{\varepsilon, \delta}\right|\right)+\left|\mathcal{R}_{\lambda}\right|\right) .
\end{aligned}
$$

Then, in the same way as in the proof of Lemma 4.1 we get the $L^{\infty}$-estimate $(4.34)$ and $\mathcal{R}_{u} \in \mathcal{C}^{0}(\bar{\Omega})$.

\subsection{Main term of the expansion}

In this section we assume $\Omega=\mathbb{B}(0,1)$ is a disk, $B_{\delta}$ is radially symmetric and satisfies assumption (4.5). We compute the main term of the expansion of $\mu_{\varepsilon, \delta}$ with respect to $\varepsilon$ and $\delta$ and give an estimate for the remainder. We show that $\mathcal{R}_{\lambda}$ is asymptotically smaller than $\mu_{\varepsilon, \delta}$ as $(\varepsilon, \delta) \rightarrow 0$ by writing uniform estimates for $\mathcal{R}_{\lambda}$. First of all we introduce the notations

$$
h_{\varepsilon}:=\sigma_{\varepsilon, 0}^{2}\left|\nabla u_{\varepsilon, 0}\right|^{2}, \quad h_{0}:=\sigma_{0,0}^{2}\left|\nabla u_{0,0}\right|^{2}, \quad \mathcal{R}_{h}=h_{\varepsilon}-h_{0} .
$$

According to Theorem 3.3 we have $u_{\varepsilon, 0} \in \mathcal{C}^{0}(\bar{\Omega})$ and consequently $h_{\varepsilon} \in \mathcal{C}^{1}(\Omega)$ in view of (3.5). We also clearly have $h_{0} \in \mathcal{C}^{\infty}(\Omega)$ so that $\mathcal{R}_{h} \in \mathcal{C}^{1}(\Omega)$. In view of the definitions of $I_{\delta}^{ \pm}$we have

$$
\int_{I_{\delta}^{+}} r-r^{*} \geq 0 \text { and }-\int_{I_{\delta}^{-}} r-r^{*} \geq 0 .
$$

Therefore, introducing the notation $l(\delta)$ we have

$$
l(\delta):=\int_{I_{\delta}^{+}}\left(r-r^{*}\right)-\int_{I_{\delta}^{-}}\left(r-r^{*}\right) \geq 0 .
$$

With a slight abuse of notation, in what follows we use the notation $h_{\varepsilon}(r), h_{0}(r)$ and $\mathcal{R}_{h}(r)$, where $r=|x|$, for the one-dimensional functions associated with $h_{\varepsilon}, h_{0}$ and $\mathcal{R}_{h}$.

Theorem 4.3. We have the expansion

$$
\mu_{\varepsilon, \delta}=\varepsilon l(\delta) \alpha^{-2} h_{0}^{\prime}\left(r^{*}\right)+\mathcal{R}_{\mu} \text { as }(\varepsilon, \delta) \rightarrow 0,
$$

where $\mathcal{R}_{\mu}=\mathcal{O}\left(\left(\varepsilon^{1 / 2}+\delta\right) \varepsilon l(\delta)\right)$. 
Proof. First we introduce the decomposition

$$
\mu_{\varepsilon, \delta}=\mu_{\varepsilon, \delta}^{1}+\mu_{\varepsilon, \delta}^{2}
$$

with

$$
\begin{aligned}
\mu_{\varepsilon, \delta}^{1} & :=\int_{\Omega} \frac{\sigma_{\varepsilon, \delta}-\sigma_{\varepsilon, 0}}{\sigma_{\varepsilon, \delta} \sigma_{\varepsilon, 0}} h_{0}, \\
\mu_{\varepsilon, \delta}^{2} & :=\int_{\Omega} \frac{\sigma_{\varepsilon, \delta}-\sigma_{\varepsilon, 0}}{\sigma_{\varepsilon, \delta} \sigma_{\varepsilon, 0}} \mathcal{R}_{h} .
\end{aligned}
$$

To get the expansion of $\mu_{\varepsilon, \delta}^{1}$, we use a Taylor expansion of $h_{0} \in \mathcal{C}^{\infty}(0,1)$ about $r=r^{*}$ :

$$
h_{0}(r)=h_{0}\left(r^{*}\right)+h_{0}^{\prime}\left(r^{*}\right)\left(r-r^{*}\right)+\frac{h_{0}^{\prime \prime}(\zeta)}{2}\left(r-r^{*}\right)^{2},
$$

where $\zeta \in\left[r^{*}, r\right]$ or $\zeta \in\left[r, r^{*}\right]$ depending on the sign of $r-r^{*}$. Note that $\zeta$ actually depends on $r$. Plugging the Taylor expansion in $\mu_{\varepsilon, \delta}^{1}$ we consider the following expansion

$$
\mu_{\varepsilon, \delta}^{1}=\mu_{\varepsilon, \delta}^{11}+\mu_{\varepsilon, \delta}^{12}+\mu_{\varepsilon, \delta}^{13}
$$

with

$$
\begin{aligned}
\mu_{\varepsilon, \delta}^{11} & :=h_{0}\left(r^{*}\right) \int_{\Omega} \frac{\sigma_{\varepsilon, \delta}-\sigma_{\varepsilon, 0}}{\sigma_{\varepsilon, \delta} \sigma_{\varepsilon, 0}}, \\
\mu_{\varepsilon, \delta}^{12} & :=h_{0}^{\prime}\left(r^{*}\right) \int_{\Omega} \frac{\left(\sigma_{\varepsilon, \delta}-\sigma_{\varepsilon, 0}\right)\left(r-r^{*}\right)}{\sigma_{\varepsilon, \delta} \sigma_{\varepsilon, 0}}, \\
\mu_{\varepsilon, \delta}^{13} & :=\int_{\Omega} \frac{\left(\sigma_{\varepsilon, \delta}-\sigma_{\varepsilon, 0}\right) h_{0}^{\prime \prime}(\zeta)\left(r-r^{*}\right)^{2}}{2 \sigma_{\varepsilon, \delta} \sigma_{\varepsilon, 0}} .
\end{aligned}
$$

We observe that $\sigma_{\varepsilon, 0} \sigma_{\varepsilon, \delta}=\alpha(\alpha+\varepsilon)$ in $I_{\delta}^{+} \cup I_{\delta}^{-}$so that in view of $\left|I_{\delta}^{+}\right|=\left|I_{\delta}^{-}\right|$we immediately get

$$
\mu_{\varepsilon, \delta}^{11}=0 .
$$

Now we turn to the computation of $\mu_{\varepsilon, \delta}^{12}$. We have

$$
\begin{aligned}
\mu_{\varepsilon, \delta}^{12} & =\varepsilon h_{0}^{\prime}\left(r^{*}\right)\left(\int_{I_{\delta}^{+}} \frac{r-r^{*}}{\alpha(\alpha+\varepsilon)}-\int_{I_{\delta}^{-}} \frac{r-r^{*}}{\alpha(\alpha+\varepsilon)}\right)=\varepsilon \frac{h_{0}^{\prime}\left(r^{*}\right)}{\alpha(\alpha+\varepsilon)} l(\delta) \\
& =\varepsilon \alpha^{-2} h_{0}^{\prime}\left(r^{*}\right) l(\delta)+\mathcal{R}_{12}(\varepsilon, \delta),
\end{aligned}
$$

with

$$
\mathcal{R}_{12}(\varepsilon, \delta)=\mathcal{O}\left(\varepsilon^{2} l(\delta)\right)
$$

It holds

$$
\left|\mu_{\varepsilon, \delta}^{13}\right| \leq \frac{\varepsilon}{2 \alpha(\alpha+\varepsilon)} \sup _{r \in\left[r^{*}-\delta, r^{*}+\delta\right]}\left|h_{0}^{\prime \prime}(\zeta)\left(r-r^{*}\right)\right| \int_{\Omega}\left|\left(\chi_{I_{\delta}^{+}}-\chi_{I_{\delta}^{-}}\right)\left(r-r^{*}\right)\right| .
$$

Since $\left(\chi_{I_{\delta}^{+}}-\chi_{I_{\delta}^{-}}\right)\left(r-r^{*}\right) \geq 0$ we observe that

$$
\int_{\Omega}\left|\left(\chi_{I_{\delta}^{+}}-\chi_{I_{\delta}^{-}}\right)\left(r-r^{*}\right)\right|=\int_{\Omega}\left(\chi_{I_{\delta}^{+}}-\chi_{I_{\delta}^{-}}\right)\left(r-r^{*}\right)=l(\delta) .
$$


We also have, since $h_{0} \in C^{\infty}(0,1)$

$$
\sup _{r \in\left[r^{*}-\delta, r^{*}+\delta\right]}\left|h_{0}^{\prime \prime}(\zeta)\left(r-r^{*}\right)\right| \leq c \delta
$$

Finally this yields using (4.11)

$$
\left|\mu_{\varepsilon, \delta}^{13}\right| \leq \operatorname{c\varepsilon \delta l}(\delta)
$$

Next, since $\mathcal{R}_{h} \in \mathcal{C}^{1}(0,1)$ we consider the expansion

$$
\mathcal{R}_{h}(r)=\mathcal{R}_{h}\left(r^{*}\right)+\mathcal{R}_{h}^{\prime}(\zeta)\left(r-r^{*}\right)
$$

where $\zeta \in\left[r^{*}, r\right]$ or $\zeta \in\left[r, r^{*}\right]$ depending on the sign of $r-r^{*}$. Note that $\zeta$ depends on $r$. Introduce

$$
\mu_{\varepsilon, \delta}^{2}=\mu_{\varepsilon, \delta}^{21}+\mu_{\varepsilon, \delta}^{22}
$$

with

$$
\begin{aligned}
\mu_{\varepsilon, \delta}^{21} & :=\mathcal{R}_{h}\left(r^{*}\right) \int_{\Omega} \frac{\sigma_{\varepsilon, \delta}-\sigma_{\varepsilon, 0}}{\sigma_{\varepsilon, \delta} \sigma_{\varepsilon, 0}} \\
\mu_{\varepsilon, \delta}^{22} & :=\int_{\Omega} \frac{\left(\sigma_{\varepsilon, \delta}-\sigma_{\varepsilon, 0}\right) \mathcal{R}_{h}^{\prime}(\zeta)\left(r-r^{*}\right)}{\sigma_{\varepsilon, \delta} \sigma_{\varepsilon, 0}} .
\end{aligned}
$$

We clearly have, for the same reasons for which we had $\mu_{\varepsilon, \delta}^{11}=0$, that

$$
\mu_{\varepsilon, \delta}^{21}=0 .
$$

For $\mu_{\varepsilon, \delta}^{22}$ we use

$$
\begin{aligned}
\left|\mu_{\varepsilon, \delta}^{22}\right| & \leq \frac{\varepsilon}{\alpha(\alpha+\varepsilon)} \sup _{r \in\left[r^{*}-\delta, r^{*}+\delta\right]}\left|\mathcal{R}_{h}^{\prime}(\zeta)\right| \int_{\Omega}\left|\left(\chi_{I_{\delta}^{+}}-\chi_{I_{\delta}^{-}}\right)\left(r-r^{*}\right)\right| \\
& \leq \frac{\varepsilon}{\alpha(\alpha+\varepsilon)} l(\delta) \sup _{r \in\left[r^{*}-\delta, r^{*}+\delta\right]}\left|\mathcal{R}_{h}^{\prime}(\zeta)\right| .
\end{aligned}
$$

We now proceed to estimate $\sup _{r \in\left[r^{*}-\delta, r^{*}+\delta\right]}\left|\mathcal{R}_{h}^{\prime}(\zeta)\right|$. First we have

$$
\mathcal{R}_{h}=\left(\sigma_{\varepsilon, 0}\right)^{2}\left|\nabla u_{\varepsilon, 0}\right|^{2}-\left(\sigma_{0,0}\right)^{2}\left|\nabla u_{0,0}\right|^{2}=g_{\varepsilon} \tilde{g}_{\varepsilon}
$$

with

$$
\begin{aligned}
& g_{\varepsilon}:=\sigma_{\varepsilon, 0} \nabla u_{\varepsilon, 0}-\sigma_{0,0} \nabla u_{0,0}, \\
& \tilde{g}_{\varepsilon}:=\sigma_{\varepsilon, 0} \nabla u_{\varepsilon, 0}+\sigma_{0,0} \nabla u_{0,0} .
\end{aligned}
$$

Next we prove that

$$
\left\|\mathcal{R}_{h}^{\prime}\right\|_{L^{\infty}\left(r^{*} / 2,1\right)} \leq c \sqrt{\varepsilon}
$$

We have $\mathcal{R}_{h}^{\prime}=g_{\varepsilon}^{\prime} \tilde{g}_{\varepsilon}+g_{\varepsilon} \tilde{g}_{\varepsilon}^{\prime}$. We have seen in (3.7) that $g_{\varepsilon}$ satisfies the equation

$$
\frac{\mathrm{d}}{\mathrm{d} r}\left[r^{d-1} g_{\varepsilon}(r)\right]=-r^{d-1}\left(\lambda_{\varepsilon} \bar{u}_{\varepsilon}(r)-\lambda_{0} \bar{u}_{0}(r)\right)
$$

which gives

$$
r^{d-1} g_{\varepsilon}^{\prime}(r)=(d-1) r^{d-2} g_{\varepsilon}(r)-r^{d-1}\left(\lambda_{\varepsilon} \bar{u}_{\varepsilon}(r)-\lambda_{0} \bar{u}_{0}(r)\right) .
$$

Since $\left\|g_{\varepsilon}\right\|_{L^{\infty}(0,1)} \leq c \sqrt{\varepsilon}$ and $\left\|\lambda_{\varepsilon} \bar{u}_{\varepsilon}(r)-\lambda_{0} \bar{u}_{0}(r)\right\|_{L^{\infty}(0,1)} \leq c \sqrt{\varepsilon}$ in view of Theorem 3.3, equation (4.46) yields

$$
\left\|g_{\varepsilon}^{\prime}\right\|_{L^{\infty}\left(r^{*} / 2,1\right)} \leq c \sqrt{\varepsilon}
$$


Using Theorem 3.3 we also get $\left\|\tilde{g}_{\varepsilon}\right\|_{L^{\infty}(0,1)} \leq c$ and we deduce in a similar way $\left\|\tilde{g}_{\varepsilon}^{\prime}\right\|_{L^{\infty}\left(r^{*} / 2,1\right)} \leq c$. Combining these results with $\mathcal{R}_{h}^{\prime}=g_{\varepsilon}^{\prime} \tilde{g}_{\varepsilon}+g_{\varepsilon} \tilde{g}_{\varepsilon}^{\prime}$ we get

$$
\left\|\mathcal{R}_{h}^{\prime}\right\|_{L^{\infty}\left(r^{*} / 2,1\right)} \leq c \sqrt{\varepsilon}
$$

Inserting this result into (4.45) we obtain

$$
\left|\mu_{\varepsilon, \delta}^{22}\right| \leq c \varepsilon^{3 / 2} l(\delta)
$$

Finally, considering the decomposition

$$
\mu_{\varepsilon, \delta}=\mu_{\varepsilon, \delta}^{11}+\mu_{\varepsilon, \delta}^{12}+\mu_{\varepsilon, \delta}^{13}+\mu_{\varepsilon, \delta}^{21}+\mu_{\varepsilon, \delta}^{22}
$$

and gathering (4.41), (4.42), (4.43), (4.44), (4.47) we have proved (4.36).

In view of (4.36) we need to show that $\varepsilon \alpha^{-2} h_{0}^{\prime}\left(r^{*}\right) l(\delta) \geq 0$ for all $\varepsilon>0, \delta>0$ to prove the main result. Since $\alpha>0$ and $l(\delta) \geq 0$ one should prove that $h_{0}^{\prime}\left(r^{*}\right)>0$. This is given in the following

Lemma 4.4. If $m<\bar{m}$ then $h_{0}^{\prime}\left(r^{*}\right)>0$.

Proof. We have $h_{0}=\sigma_{0,0}^{2}\left|\nabla u_{0,0}\right|^{2} \in \mathcal{C}^{\infty}(\Omega)$ when $\Omega=\mathbb{B}(0,1)$. We compute

$$
h_{0}^{\prime}=2 \sigma_{0,0}^{2} \bar{u}_{0,0}^{\prime} \bar{u}_{0,0}^{\prime \prime} \text {. }
$$

We have seen in Section 3 that $u_{0,0}$ is given by the Bessel functions (3.3) and (3.4) for $d=2$ and $d=3$, respectively. It is a standard result that $\bar{u}_{0,0}^{\prime}$ is negative, decreasing on $\left[0, r_{d}^{1}\right]$ and increasing on $\left[r_{d}^{1}, 1\right]$, where $0<r_{d}^{1}<1$ is defined in Figure 1. Thus we get

$$
\begin{aligned}
& h_{0}^{\prime}(r)>0 \text { for } r \in\left[0, r_{d}^{1}[,\right. \\
& h_{0}^{\prime}(r)=0 \text { for } r=r_{d}^{1}, \\
& \left.\left.h_{0}^{\prime}(r)<0 \text { for } r \in\right] r_{d}^{1}, 1\right] .
\end{aligned}
$$

Thanks to the assumption $m<\bar{m}$ we get $r^{*}<r_{d}^{0}<r_{d}^{1}$ which completes the proof using (4.48).

\subsection{Estimate of the remainder}

In this section we give the estimate with respect to $(\varepsilon, \delta)$ for the remainder $\mathcal{R}_{\lambda}$ in expansion (4.19).

Theorem 4.5. We have the estimate

$$
\left|\mathcal{R}_{\lambda}\right| \leq c \varepsilon^{2} l(\delta)\left(\left|I_{\delta}^{+}\right|^{1 / 2}+l(\delta)\right) \text { as }(\varepsilon, \delta) \rightarrow 0
$$

where $c>0$ is independent of $(\varepsilon, \delta)$.

Proof. Step 1. First of all we define the following functions

$$
F_{0}:=\sigma_{\varepsilon, 0} \nabla u_{\varepsilon, 0}, \quad F_{1}:=\sigma_{\varepsilon, 0} \nabla w_{\varepsilon, \delta}, \quad F_{2}:=\sigma_{\varepsilon, \delta} \nabla \mathcal{R}_{u} .
$$

For the sake of simplicity we will denote indistinctly $F_{0}, F_{1}, F_{2}$ and $u_{\varepsilon, 0}, w_{\varepsilon, \delta}, \mathcal{R}_{u}$ for the functions taking arguments in $\mathbb{R}^{d}$ or for their one-dimensional counterparts. We decompose $F_{0}, F_{1}$ and $F_{2}$ into a smooth and a nonsmooth part. In view of $(4.22)-(4.23)$ we have

$$
-r^{1-d} \frac{\mathrm{d}}{\mathrm{d} r}\left[r^{d-1} F_{1}(r)\right]=r^{1-d} \frac{\mathrm{d}}{\mathrm{d} r}\left[r^{d-1} \frac{\left(\sigma_{\varepsilon, \delta}-\sigma_{\varepsilon, 0}\right) \sigma_{\varepsilon, 0}}{\sigma_{\varepsilon, \delta}} u_{\varepsilon, 0}^{\prime}\right]+\mu_{\varepsilon, \delta} u_{\varepsilon, 0}+\lambda_{\varepsilon, 0} w_{\varepsilon, \delta},
$$


Dividing by $r^{1-d}$ and integrating from 0 to $r$ we get

$$
-r^{d-1} F_{1}(r)=r^{d-1} \frac{\left(\sigma_{\varepsilon, \delta}-\sigma_{\varepsilon, 0}\right) \sigma_{\varepsilon, 0}}{\sigma_{\varepsilon, \delta}} u_{\varepsilon, 0}^{\prime}+\int_{0}^{r} t^{d-1}\left(\mu_{\varepsilon, \delta} u_{\varepsilon, 0}(t)+\lambda_{\varepsilon, 0} w_{\varepsilon, \delta}(t)\right) \mathrm{d} t,
$$

where we have used $u_{\varepsilon, 0}^{\prime}(0)=0$ and $\lim _{t \rightarrow 0} t^{d-1} F_{1}(t)=0$. This yields

$$
F_{1}(r)=-\frac{\left(\sigma_{\varepsilon, \delta}-\sigma_{\varepsilon, 0}\right) \sigma_{\varepsilon, 0}}{\sigma_{\varepsilon, \delta}} u_{\varepsilon, 0}^{\prime}+F_{11}(r)
$$

where

$$
F_{11}(r)=-r^{1-d} \int_{0}^{r} t^{d-1}\left(\mu_{\varepsilon, \delta} u_{\varepsilon, 0}(t)+\lambda_{\varepsilon, 0} w_{\varepsilon, \delta}(t)\right) \mathrm{d} t
$$

In a similar way, we get

$$
\begin{gathered}
F_{2}(r)=-\left(\sigma_{\varepsilon, \delta}-\sigma_{\varepsilon, 0}\right)\left[\frac{\left(\sigma_{\varepsilon, \delta}-\sigma_{\varepsilon, 0}\right)}{\sigma_{\varepsilon, \delta}} u_{\varepsilon, 0}^{\prime}+w_{\varepsilon, \delta}^{\prime}\right]+F_{21}(r), \\
F_{21}(r)=-r^{1-d} \int_{0}^{r} t^{d-1}\left(\mu_{\varepsilon, \delta} w_{\varepsilon, \delta}+\mathcal{R}_{\lambda} u_{\varepsilon, \delta}+\lambda_{\varepsilon, \delta} \mathcal{R}_{u}\right) \mathrm{d} t .
\end{gathered}
$$

and

$$
F_{0}(r)=-r^{1-d} \int_{0}^{r} t^{d-1}\left(\lambda_{\varepsilon, 0} u_{\varepsilon, 0}\right) \mathrm{d} t .
$$

In (4.54) we can replace $w_{\varepsilon, \delta}^{\prime}$ using (4.52). After simplification this yields

$$
F_{2}(r)=-\frac{\left(\sigma_{\varepsilon, \delta}-\sigma_{\varepsilon, 0}\right)}{\sigma_{\varepsilon, 0}} F_{11}(r)+F_{21}(r),
$$

Step 2. Next we decompose $\mathcal{R}_{\lambda}^{2}$ and $\mathcal{R}_{\lambda}^{3}$ in the following way

$$
\mathcal{R}_{\lambda}^{2}=\mathcal{R}_{\lambda}^{21}+\mathcal{R}_{\lambda}^{22}+\mathcal{R}_{\lambda}^{23} \text { and } \mathcal{R}_{\lambda}^{3}=\mathcal{R}_{\lambda}^{31}+\mathcal{R}_{\lambda}^{32}+\mathcal{R}_{\lambda}^{33}
$$

where

$$
\begin{aligned}
& \mathcal{R}_{\lambda}^{21}:=\int_{\Omega} \frac{\left(\sigma_{\varepsilon, \delta}-\sigma_{\varepsilon, 0}\right)^{2}}{\sigma_{\varepsilon, \delta} \sigma_{\varepsilon, 0}^{2}}\left(F_{0}\right)^{2}, \quad \mathcal{R}_{\lambda}^{22}:=\int_{\Omega} \frac{\left(\sigma_{\varepsilon, \delta}-\sigma_{\varepsilon, 0}\right)^{2}}{\sigma_{\varepsilon, \delta} \sigma_{\varepsilon, 0}^{2}} F_{0} F_{1}, \\
& \mathcal{R}_{\lambda}^{23}:=\int_{\Omega} \frac{\left(\sigma_{\varepsilon, \delta}-\sigma_{\varepsilon, 0}\right)^{2}}{\sigma_{\varepsilon, \delta}^{2} \sigma_{\varepsilon, 0}} F_{0} F_{2},
\end{aligned}
$$

and

$$
\begin{aligned}
& \mathcal{R}_{\lambda}^{31}:=\int_{\Omega} \frac{\sigma_{\varepsilon, \delta}-\sigma_{\varepsilon, 0}}{\sigma_{\varepsilon, 0}^{2}} F_{0} F_{1}, \quad \mathcal{R}_{\lambda}^{32}:=\int_{\Omega} \frac{\sigma_{\varepsilon, \delta}-\sigma_{\varepsilon, 0}}{\sigma_{\varepsilon, 0}^{2}}\left(F_{1}\right)^{2}, \\
& \mathcal{R}_{\lambda}^{33}:=\int_{\Omega} \frac{\sigma_{\varepsilon, \delta}-\sigma_{\varepsilon, 0}}{\sigma_{\varepsilon, 0} \sigma_{\varepsilon, \delta}} F_{1} F_{2} .
\end{aligned}
$$

Now we compute using (4.52):

$$
\begin{aligned}
\mathcal{R}_{\lambda}^{21}+\mathcal{R}_{\lambda}^{31} & =\int_{\Omega} \frac{\left(\sigma_{\varepsilon, \delta}-\sigma_{\varepsilon, 0}\right)^{2}}{\sigma_{\varepsilon, \delta} \sigma_{\varepsilon, 0}^{2}}\left(F_{0}\right)^{2}+\int_{\Omega} \frac{\sigma_{\varepsilon, \delta}-\sigma_{\varepsilon, 0}}{\sigma_{\varepsilon, 0}^{2}} F_{0}\left[-\frac{\left(\sigma_{\varepsilon, \delta}-\sigma_{\varepsilon, 0}\right) \sigma_{\varepsilon, 0}}{\sigma_{\varepsilon, \delta}} \nabla u_{\varepsilon, 0}+F_{11}\right] \\
& =\int_{\Omega} \frac{\sigma_{\varepsilon, \delta}-\sigma_{\varepsilon, 0}}{\sigma_{\varepsilon, 0}^{2}} F_{0} F_{11} .
\end{aligned}
$$


Using again (4.52) we get

$$
\begin{aligned}
\mathcal{R}_{\lambda}^{22}+\mathcal{R}_{\lambda}^{32}= & \int_{\Omega} \frac{\left(\sigma_{\varepsilon, \delta}-\sigma_{\varepsilon, 0}\right)^{2}}{\sigma_{\varepsilon, \delta} \sigma_{\varepsilon, 0}^{2}} F_{0}\left[-\frac{\left(\sigma_{\varepsilon, \delta}-\sigma_{\varepsilon, 0}\right) \sigma_{\varepsilon, 0}}{\sigma_{\varepsilon, \delta}} \nabla u_{\varepsilon, 0}+F_{11}\right] \\
& +\int_{\Omega} \frac{\sigma_{\varepsilon, \delta}-\sigma_{\varepsilon, 0}}{\sigma_{\varepsilon, 0}^{2}}\left[-\frac{\left(\sigma_{\varepsilon, \delta}-\sigma_{\varepsilon, 0}\right) \sigma_{\varepsilon, 0}}{\sigma_{\varepsilon, \delta}} \nabla u_{\varepsilon, 0}+F_{11}\right]^{2} \\
= & \int_{\Omega}-\frac{\left(\sigma_{\varepsilon, \delta}-\sigma_{\varepsilon, 0}\right)^{2}}{\sigma_{\varepsilon, \delta} \sigma_{\varepsilon, 0}^{2}} F_{0} F_{11}+\frac{\sigma_{\varepsilon, \delta}-\sigma_{\varepsilon, 0}}{\sigma_{\varepsilon, 0}^{2}}\left(F_{11}\right)^{2} .
\end{aligned}
$$

For the other two terms, using (4.52) and (4.57) we get

$$
\begin{aligned}
\mathcal{R}_{\lambda}^{23}+\mathcal{R}_{\lambda}^{33} & =\int_{\Omega} \frac{\sigma_{\varepsilon, \delta}-\sigma_{\varepsilon, 0}}{\sigma_{\varepsilon, 0} \sigma_{\varepsilon, \delta}}\left[\frac{\left(\sigma_{\varepsilon, \delta}-\sigma_{\varepsilon, 0}\right)}{\sigma_{\varepsilon, \delta}} F_{0}+F_{1}\right] F_{2} \\
& =\int_{\Omega} \frac{\sigma_{\varepsilon, \delta}-\sigma_{\varepsilon, 0}}{\sigma_{\varepsilon, 0} \sigma_{\varepsilon, \delta}} F_{11}\left[-\frac{\left(\sigma_{\varepsilon, \delta}-\sigma_{\varepsilon, 0}\right)}{\sigma_{\varepsilon, 0}} F_{11}+F_{21}\right] \\
& =\int_{\Omega} \frac{\sigma_{\varepsilon, \delta}-\sigma_{\varepsilon, 0}}{\sigma_{\varepsilon, 0} \sigma_{\varepsilon, \delta}} F_{11} F_{21}-\frac{\left(\sigma_{\varepsilon, \delta}-\sigma_{\varepsilon, 0}\right)^{2}}{\sigma_{\varepsilon, 0}^{2} \sigma_{\varepsilon, \delta}}\left(F_{11}\right)^{2} .
\end{aligned}
$$

We proceed with further simplifications by summing all terms:

$$
\begin{aligned}
\mathcal{R}_{\lambda}^{2}+\mathcal{R}_{\lambda}^{3}= & \mathcal{R}_{\lambda}^{21}+\mathcal{R}_{\lambda}^{31}+\mathcal{R}_{\lambda}^{22}+\mathcal{R}_{\lambda}^{32}+\mathcal{R}_{\lambda}^{23}+\mathcal{R}_{\lambda}^{33} \\
= & \int_{\Omega}\left[\frac{\sigma_{\varepsilon, \delta}-\sigma_{\varepsilon, 0}}{\sigma_{\varepsilon, 0}^{2}}-\frac{\left(\sigma_{\varepsilon, \delta}-\sigma_{\varepsilon, 0}\right)^{2}}{\sigma_{\varepsilon, \delta} \sigma_{\varepsilon, 0}^{2}}\right] F_{0} F_{11} \\
& +\int_{\Omega}\left[\frac{\sigma_{\varepsilon, \delta}-\sigma_{\varepsilon, 0}}{\sigma_{\varepsilon, 0}^{2}}-\frac{\left(\sigma_{\varepsilon, \delta}-\sigma_{\varepsilon, 0}\right)^{2}}{\sigma_{\varepsilon, 0}^{2} \sigma_{\varepsilon, \delta}}\right]\left(F_{11}\right)^{2}+\int_{\Omega} \frac{\sigma_{\varepsilon, \delta}-\sigma_{\varepsilon, 0}}{\sigma_{\varepsilon, 0} \sigma_{\varepsilon, \delta}} F_{11} F_{21} \\
= & \int_{\Omega} \frac{\sigma_{\varepsilon, \delta}-\sigma_{\varepsilon, 0}}{\sigma_{\varepsilon, \delta} \sigma_{\varepsilon, 0}}\left(F_{0} F_{11}+\left(F_{11}\right)^{2}+F_{11} F_{21}\right) .
\end{aligned}
$$

We have obtained

$$
\mathcal{R}_{\lambda}^{2}+\mathcal{R}_{\lambda}^{3}=\int_{\Omega} \frac{\sigma_{\varepsilon, \delta}-\sigma_{\varepsilon, 0}}{\sigma_{\varepsilon, \delta} \sigma_{\varepsilon, 0}} F
$$

with

$$
F:=F_{0} F_{11}+\left(F_{11}\right)^{2}+F_{11} F_{21}=F_{11}\left(F_{0}+F_{11}+F_{21}\right) .
$$

Step 3. Since the functions $u_{\varepsilon, 0}, w_{\varepsilon, \delta}, u_{\varepsilon, \delta}$ and $\mathcal{R}_{u}$ are in $\mathcal{C}^{0}(\bar{\Omega})$ due to Theorem 3.3, Lemmas 4.1 and 4.2 , we get $F_{0}, F_{11}, F_{21} \in \mathcal{C}^{1}(0,1)$ and a fortiori $F \in \mathcal{C}^{1}(0,1)$. Therefore it is possible to consider the Taylor expansion

$$
F(r)=F\left(r^{*}\right)+F^{\prime}(\zeta)\left(r-r^{*}\right)
$$

where $\zeta \in\left[r^{*}, r\right]$ or $\zeta \in\left[r, r^{*}\right]$ depending on the sign of $r-r^{*}$. Replacing in the previous expression we get

$$
\mathcal{R}_{\lambda}^{2}+\mathcal{R}_{\lambda}^{3}=F\left(r^{*}\right) \int_{\Omega} \frac{\sigma_{\varepsilon, \delta}-\sigma_{\varepsilon, 0}}{\sigma_{\varepsilon, \delta} \sigma_{\varepsilon, 0}}+\int_{\Omega} \frac{\sigma_{\varepsilon, \delta}-\sigma_{\varepsilon, 0}}{\sigma_{\varepsilon, \delta} \sigma_{\varepsilon, 0}} F^{\prime}(\zeta)\left(r-r^{*}\right) .
$$

As already observed above we have

$$
\int_{\Omega} \frac{\sigma_{\varepsilon, \delta}-\sigma_{\varepsilon, 0}}{\sigma_{\varepsilon, \delta} \sigma_{\varepsilon, 0}}=0
$$


and

$$
\left|\int_{\Omega} \frac{\sigma_{\varepsilon, \delta}-\sigma_{\varepsilon, 0}}{\sigma_{\varepsilon, \delta} \sigma_{\varepsilon, 0}} F^{\prime}(\zeta)\left(r-r^{*}\right)\right| \leq \frac{\varepsilon l(\delta)}{\alpha(\alpha+\varepsilon)} \sup _{r \in\left[r^{*}-\delta, r^{*}+\delta\right]}\left|F^{\prime}(r)\right| \leq c .
$$

We give now an estimate for the supremum in (4.58). We have

$$
F^{\prime}=F_{11}^{\prime}\left(F_{0}+F_{11}+F_{21}\right)+F_{11}\left(F_{0}^{\prime}+F_{11}^{\prime}+F_{21}^{\prime}\right)
$$

Using (4.56) we get

$$
\sup _{r \in\left[r^{*}-\delta, r^{*}+\delta\right]}\left|F_{0}(r)\right| \leq c
$$

and

$$
F_{0}^{\prime}=r^{-1}(1-d) F_{0}-\lambda_{\varepsilon, 0} u_{\varepsilon, 0}
$$

so that

$$
\sup _{r \in\left[r^{*}-\delta, r^{*}+\delta\right]}\left|F_{0}^{\prime}(r)\right| \leq c
$$

According to (4.53) we get

$$
F_{11}^{\prime}=r^{-1}(1-d) F_{11}-\left(\mu_{\varepsilon, \delta} u_{\varepsilon, 0}+\lambda_{\varepsilon, 0} w_{\varepsilon, \delta}\right)
$$

so that

$$
\sup _{r \in\left[r^{*}-\delta, r^{*}+\delta\right]}\left|F_{11}^{\prime}(r)\right| \leq c\left(\sup _{r \in\left[r^{*}-\delta, r^{*}+\delta\right]}\left|F_{11}(r)\right|+\left|\mu_{\varepsilon, \delta}\right|+\left\|w_{\varepsilon, \delta}\right\|_{L^{\infty}(0,1)}\right),
$$

where we have used the fact that $u_{\varepsilon, 0}$ is uniformly bounded on $\Omega$ independently of $\varepsilon$ and $\lambda_{\varepsilon, 0} \leq c$ as well. Using expression (4.53) we get

$$
\sup _{r \in\left[r^{*}-\delta, r^{*}+\delta\right]}\left|F_{11}(r)\right| \leq c\left(\left|\mu_{\varepsilon, \delta}\right|+\left\|w_{\varepsilon, \delta}\right\|_{L^{\infty}(0,1)}\right)
$$

and consequently

$$
\sup _{r \in\left[r^{*}-\delta, r^{*}+\delta\right]}\left|F_{11}^{\prime}(r)\right| \leq c\left(\left|\mu_{\varepsilon, \delta}\right|+\left\|w_{\varepsilon, \delta}\right\|_{L^{\infty}(0,1)}\right) .
$$

Using Lemma 4.1 we obtain

$$
\sup _{r \in\left[r^{*}-\delta, r^{*}+\delta\right]}\left|F_{11}^{\prime}(r)\right| \leq c\left(\varepsilon\left|I_{\delta}^{+}\right|^{1 / 2}+\left|\mu_{\varepsilon, \delta}\right|\right) .
$$

According to (4.55) we get

$$
\sup _{r \in\left[r^{*}-\delta, r^{*}+\delta\right]}\left|F_{21}(r)\right| \leq c\left(\left|\mu_{\varepsilon, \delta}\right|\left\|w_{\varepsilon, \delta}\right\|_{L^{\infty}(0,1)}+\left|\mathcal{R}_{\lambda}\right|+\left\|\mathcal{R}_{u}\right\|_{L^{\infty}(0,1)}\right)
$$

and

$$
F_{21}^{\prime}(r)=r^{-1}(1-d) F_{21}-\left(\mu_{\varepsilon, \delta} w_{\varepsilon, \delta}+\mathcal{R}_{\lambda} u_{\varepsilon, \delta}+\lambda_{\varepsilon, \delta} \mathcal{R}_{u}\right)
$$

so that

$$
\sup _{r \in\left[r^{*}-\delta, r^{*}+\delta\right]}\left|F_{21}^{\prime}(r)\right| \leq c\left(\left|\mu_{\varepsilon, \delta}\right|\left\|w_{\varepsilon, \delta}\right\|_{L^{\infty}(0,1)}+\left|\mathcal{R}_{\lambda}\right|+\left\|\mathcal{R}_{u}\right\|_{L^{\infty}(0,1)}\right) .
$$

Gathering the previous results we obtain

$$
\left.\sup _{r \in\left[r^{*}-\delta, r^{*}+\delta\right]}\left|F^{\prime}(r)\right| \leq c\left(\left|\mu_{\varepsilon, \delta}\right|+\left\|w_{\varepsilon, \delta}\right\|_{L^{\infty}(0,1)}\right)\left(c+\left|\mathcal{R}_{\lambda}\right|+\left\|\mathcal{R}_{u}\right\|_{L^{\infty}(0,1)}\right)\right) .
$$

Plugging this result into (4.58) we obtain

$$
\left.\left|\int_{\Omega} \frac{\sigma_{\varepsilon, \delta}-\sigma_{\varepsilon, 0}}{\sigma_{\varepsilon, \delta} \sigma_{\varepsilon, 0}} F^{\prime}(\zeta)\left(r-r^{*}\right)\right| \leq \varepsilon l(\delta) c\left(\left|\mu_{\varepsilon, \delta}\right|+\left\|w_{\varepsilon, \delta}\right\|_{L^{\infty}(0,1)}\right)\left(c+\left|\mathcal{R}_{\lambda}\right|+\left\|\mathcal{R}_{u}\right\|_{L^{\infty}(0,1)}\right)\right) .
$$


So finally we have obtained

$$
\left.\left|\mathcal{R}_{\lambda}^{2}+\mathcal{R}_{\lambda}^{3}\right| \leq \varepsilon l(\delta) c\left(\left|\mu_{\varepsilon, \delta}\right|+\left\|w_{\varepsilon, \delta}\right\|_{L^{\infty}(0,1)}\right)\left(c+\left|\mathcal{R}_{\lambda}\right|+\left\|\mathcal{R}_{u}\right\|_{L^{\infty}(0,1)}\right)\right) .
$$

Step 4. We have the estimate

$$
\left|\mathcal{R}_{\lambda}^{1}\right| \leq\left|\mu_{\varepsilon, \delta}\right|\left\|w_{\varepsilon, \delta}\right\|_{L^{2}(\Omega)}
$$

Gathering (4.60) and (4.59) we obtain

$$
\begin{aligned}
\left|\mathcal{R}_{\lambda}\right| & =\left|\mathcal{R}_{\lambda}^{1}+\mathcal{R}_{\lambda}^{2}+\mathcal{R}_{\lambda}^{3}\right| \\
& \left.\leq\left|\mu_{\varepsilon, \delta}\right|\left\|w_{\varepsilon, \delta}\right\|_{L^{2}(\Omega)}+\operatorname{c\varepsilon l}(\delta)\left(\left|\mu_{\varepsilon, \delta}\right|+\left\|w_{\varepsilon, \delta}\right\|_{L^{\infty}(0,1)}\right)\left(c+\left|\mathcal{R}_{\lambda}\right|+\left\|\mathcal{R}_{u}\right\|_{L^{\infty}(0,1)}\right)\right)
\end{aligned}
$$

Using (4.27), (4.34) and (4.36) we get the estimates

$$
\begin{aligned}
\left\|\mathcal{R}_{u}\right\|_{L^{\infty}(\Omega)} & \leq c \varepsilon^{2}\left(\left|I_{\delta}^{+}\right|^{1 / 2}+l(\delta)+\left|\mathcal{R}_{\lambda}\right|\right), \\
\left\|w_{\varepsilon, \delta}\right\|_{L^{\infty}(\Omega)} & \leq c \varepsilon\left(\left|I_{\delta}^{+}\right|^{1 / 2}+l(\delta)\right) .
\end{aligned}
$$

Using these estimates in (4.61) yields

$$
\left|\mathcal{R}_{\lambda}\right| \leq \varepsilon^{2} l(\delta)\left(\left|I_{\delta}^{+}\right|^{1 / 2}+l(\delta)\right)\left(c+\left|\mathcal{R}_{\lambda}\right|\right)
$$

Since $\varepsilon^{2} l(\delta)\left(\left|I_{\delta}^{+}\right|^{1 / 2}+l(\delta)\right)$ goes to zero when $(\varepsilon, \delta) \rightarrow 0$, we can move the term depending on $\left|\mathcal{R}_{\lambda}\right|$ in the right-hand side of (4.62) to the left-hand side and we obtain (4.51).

\subsection{Main result}

We can now state the main result of this paper. Let $B^{*}=\mathbb{B}\left(0, r^{*}\right)$ as given by Proposition 3.1.

Theorem 4.6. If $m<\bar{m}$ there exists $\varepsilon_{0}>0$ such that for all $B \in \mathcal{B}$ we have

$$
\lambda_{\varepsilon}\left(B^{*}\right) \leq \lambda_{\varepsilon}(B) \text { for all } 0<\varepsilon<\varepsilon_{0}
$$

and the equality occurs only when $B=B^{*}$ almost everywhere in $\Omega$.

Proof. Thanks to Theorem 2.3 we can assume $B$ is radially symmetric. In view of the proof of Corollary 3.10 , for all $\varepsilon>0$ there exists a $\delta(\varepsilon)>0$ such that

$$
\lambda_{\varepsilon}\left(B_{\delta(\varepsilon)}^{*}\right) \leq \lambda_{\varepsilon}(B)
$$

holds with $\delta(\varepsilon) \rightarrow 0$ as $\varepsilon \rightarrow 0$ and $\delta(\varepsilon)$ strictly increasing. In view of (4.19) and (4.36) we have

$$
\begin{aligned}
\lambda_{\varepsilon}\left(B_{\delta}\right)=\lambda_{\varepsilon, \delta} & =\lambda_{\varepsilon, 0}+\mu_{\varepsilon, \delta}+\mathcal{R}_{\lambda}(\varepsilon, \delta) \\
& =\lambda_{\varepsilon, 0}+\varepsilon l(\delta) \alpha^{-2} h_{0}^{\prime}\left(r^{*}\right)+\mathcal{R}_{\mu}+\mathcal{R}_{\lambda}(\varepsilon, \delta)
\end{aligned}
$$

According to Theorems 4.3 and 4.5 we have

$$
\begin{aligned}
& \left|\mathcal{R}_{\mu}\right| \leq c\left(\varepsilon^{1 / 2}+\delta\right) \varepsilon l(\delta) \\
& \left|\mathcal{R}_{\lambda}\right| \leq c \varepsilon^{2} l(\delta)\left(\left|I_{\delta}^{+}\right|^{1 / 2}+l(\delta)\right),
\end{aligned}
$$

In view of Lemma 4.4 we have $h_{0}^{\prime}\left(r^{*}\right)>0$. Therefore there exists $\varepsilon_{0}>0$ and $\delta_{0}>0$ such that for all $\varepsilon<\varepsilon_{0}$ and $\delta<\delta_{0}$ we have $\lambda_{\varepsilon}\left(B_{\delta}\right) \geq \lambda_{\varepsilon, 0}=\lambda_{\varepsilon}\left(B^{*}\right)$. In particular one can choose $B_{\delta}=B_{\delta(\varepsilon)}^{*}$ for $\varepsilon$ small enough, and using (4.63) we get

$$
\lambda_{\varepsilon}\left(B^{*}\right) \leq \lambda_{\varepsilon}\left(B_{\delta}\right)=\lambda_{\varepsilon}\left(B_{\delta(\varepsilon)}^{*}\right) \leq \lambda_{\varepsilon}(B),
$$

which gives (4.1). The case $\lambda_{\varepsilon}\left(B_{\delta(\varepsilon)}^{*}\right)=\lambda_{\varepsilon}\left(B^{*}\right)$ only occurs when $l(\delta(\varepsilon))=0$ which implies $B_{\delta(\varepsilon)}^{*}=B^{*}$ almost everywhere in view of (4.35). 


\section{Optimum of Type II}

The results of Section 4 can be extended to the case of the Type II optimum. The proof is similar, so we will only give the main results and describe the differences with Section 4 . The main difference between optima of Type I and II is that the minimizers of Type II depend on $\varepsilon$, due to the fact that there are two connected components. The proofs of the results in Section 4 should be modified accordingly.

In this section we assume $m>\bar{m}$ in order to deal with optima of Type II. Let $\delta>0$ be given, then according to Theorem 3.9 there exists $\varepsilon_{0}(\delta)$ and a radially symmetric set $B_{\delta}^{*}$ satisfying (3.29), (3.30) and (3.31) for $0<\varepsilon \leq \varepsilon_{0}(\delta)$. For such $\varepsilon$ we introduce a new set $B_{\varepsilon}^{*}=B_{\varepsilon, 0}^{*} \cup B_{\varepsilon, 1}^{*}$ with

$$
\begin{aligned}
& B_{\varepsilon, 0}^{*}=\mathbb{B}\left(0, \xi_{\varepsilon}^{0}\right), \\
& B_{\varepsilon, 1}^{*}=\mathbb{B}(0,1) \backslash \overline{\mathrm{B}\left(0, \xi_{\varepsilon}^{1}\right)},
\end{aligned}
$$

and sets $I_{\delta}^{* \pm k}, k=0,1$ such that

$$
\begin{aligned}
& I_{\delta}^{*+k}:=\left\{x \in \Omega \mid \chi_{B_{\delta, k}^{*}}(x)-\chi_{B_{\varepsilon, k}^{*}}(x)=1\right\}, \\
& I_{\delta}^{*-k}:=\left\{x \in \Omega \mid \chi_{B_{\delta, k}^{*}}(x)-\chi_{B_{\varepsilon, k}^{*}}(x)=-1\right\} .
\end{aligned}
$$

where $B_{\delta}^{*}=B_{\delta, 0}^{*} \cup B_{\delta, 1}^{*}$ as in Theorem 3.9. We also define $I_{\delta}^{* k}:=I_{\delta}^{*+k} \cup I_{\delta}^{*-k}$. The positions $\xi_{\varepsilon}^{0}$ and $\xi_{\varepsilon}^{1}$ are chosen so that

$$
\int_{\Omega} \chi_{I_{\delta}^{*+0}}-\chi_{I_{\delta}^{*-0}}=0 \text { and } \int_{\Omega} \chi_{I_{\delta}^{*+1}}-\chi_{I_{\delta}^{*-1}}=0 .
$$

Such $\xi_{\varepsilon}^{0}$ and $\xi_{\varepsilon}^{1}$ exist and are unique. Indeed $\left|I_{\delta}^{*-0}\right|$ and $\left|I_{\delta}^{*+0}\right|$ are obviously increasing and decreasing functions of $\xi_{\varepsilon}^{0}$, respectively, and continuous as well. For $\xi_{\varepsilon}^{0}$ small enough we have $\left|I_{\delta}^{*-0}\right|=0$ and for $\xi_{\varepsilon}^{0}$ large enough we have $\left|I_{\delta}^{*+0}\right|=0$ so there exists a unique $\xi_{\varepsilon}^{0}$ such that $\left|I_{\delta}^{*-0}\right|=\left|I_{\delta}^{*+0}\right|$. Due to the volume constraint $\left|B_{\varepsilon}^{*}\right|=m$ and since $B_{\varepsilon}^{*}$ has two connected components, $\xi_{\varepsilon}^{1}$ is uniquely determined by the choice of $\xi_{\varepsilon}^{0}$ and we have

$$
\begin{aligned}
m & =\left|B_{\delta, 0}^{*}\right|+\left|B_{\delta, 1}^{*}\right| \\
& =\left|I_{\delta}^{*+0}\right|+\left|B_{\varepsilon, 0}^{*} \cap B_{\delta, 0}^{*}\right|+\left|I_{\delta}^{*+1}\right|+\left|B_{\varepsilon, 1}^{*} \cap B_{\delta, 1}^{*}\right| \\
& =\left|I_{\delta}^{*+0}\right|+\left|B_{\varepsilon, 0}^{*}\right|-\left|I_{\delta}^{*-0}\right|+\left|I_{\delta}^{*+1}\right|+\left|B_{\varepsilon, 1}^{*} \cap B_{\delta, 1}^{*}\right| \\
& =\left|B_{\varepsilon, 0}^{*}\right|+\left|I_{\delta}^{*+1}\right|+\left|B_{\varepsilon, 1}^{*} \cap B_{\delta, 1}^{*}\right|
\end{aligned}
$$

which yields

$$
\left|I_{\delta}^{*+1}\right|=m-\left|B_{\varepsilon, 0}^{*}\right|-\left|B_{\varepsilon, 1}^{*} \cap B_{\delta, 1}^{*}\right|=\left|B_{\varepsilon, 1}^{*}\right|-\left|B_{\varepsilon, 1}^{*} \cap B_{\delta, 1}^{*}\right|=\left|I_{\delta}^{*-1}\right| .
$$

Thus $\xi_{\varepsilon}^{0}$ and $\xi_{\varepsilon}^{1}$ can be chosen in a unique way so that (5.5) occurs. In addition since $B_{\delta}^{*}$ satisfies (3.29), (3.30) we have

$$
\left|\xi_{\varepsilon}^{0}-\xi^{0}\right| \leq \delta \text { and }\left|\xi_{\varepsilon}^{1}-\xi^{1}\right| \leq \delta .
$$

Following (3.29)-(3.30) we consider any radially symmetric set $B_{\delta}=B_{\delta, 0} \cup B_{\delta, 1}$ such that

$$
\begin{aligned}
\mathbb{B}\left(0, \xi^{0}-\delta\right) & \subset B_{\delta, 0} \subset \mathbb{B}\left(0, \xi^{0}+\delta\right), \\
\mathbb{B}(0,1) \backslash \overline{\mathrm{B}\left(0, \xi^{1}+\delta\right)} & \subset B_{\delta, 1} \subset \mathbb{B}(0,1) \backslash \overline{\mathrm{B}\left(0, \xi^{1}-\delta\right),}
\end{aligned}
$$

and satisfying

$$
\int_{\Omega} \chi_{I_{\delta}^{+0}}-\chi_{I_{\delta}^{-0}}=0 \text { and } \int_{\Omega} \chi_{I_{\delta}^{+1}}-\chi_{I_{\delta}^{-1}}=0 .
$$


where $I_{\delta}^{ \pm k}, k=0,1$ are defined by

$$
\begin{aligned}
& I_{\delta}^{+k}:=\left\{x \in \Omega \mid \chi_{B_{\delta, k}}(x)-\chi_{B_{\varepsilon, k}^{*}}(x)=1\right\}, \\
& I_{\delta}^{-k}:=\left\{x \in \Omega \mid \chi_{B_{\delta, k}}(x)-\chi_{B_{\varepsilon, k}^{*}}(x)=-1\right\} .
\end{aligned}
$$

We also define $I_{\delta}^{k}:=I_{\delta}^{+k} \cup I_{\delta}^{-k}$. Due to assumptions (5.7)-(5.8) and to (5.6) we have

$$
\begin{array}{lll}
I_{\delta}^{+0} \subset \mathbb{B}\left(0, \xi_{\varepsilon}^{0}+2 \delta\right) \backslash \mathbb{B}\left(0, \xi_{\varepsilon}^{0}\right), & & I_{\delta}^{+1} \subset \mathbb{B}\left(0, \xi_{\varepsilon}^{1}\right) \backslash \mathbb{B}\left(0, \xi_{\varepsilon}^{1}-2 \delta\right), \\
I_{\delta}^{-0} \subset \mathbb{B}\left(0, \xi_{\varepsilon}^{0}\right) \backslash \mathbb{B}\left(0, \xi_{\varepsilon}^{0}-2 \delta\right), & & I_{\delta}^{-1} \subset \mathbb{B}\left(0, \xi_{\varepsilon}^{1}+2 \delta\right) \backslash \mathbb{B}\left(0, \xi_{\varepsilon}^{1}\right) .
\end{array}
$$

As in Section 4, we aim at obtaining an asymptotic expansion of $\lambda_{\varepsilon, \delta}=\lambda_{\varepsilon}\left(B_{\delta}\right)$ with respect to $(\varepsilon, \delta)$ of the type

$$
\begin{aligned}
u_{\varepsilon, \delta} & =u_{\varepsilon, 0}+w_{\varepsilon, \delta}+\mathcal{R}_{u}(\varepsilon, \delta), \\
\lambda_{\varepsilon, \delta} & =\lambda_{\varepsilon, 0}+\mu_{\varepsilon, \delta}+\mathcal{R}_{\lambda}(\varepsilon, \delta),
\end{aligned}
$$

We introduce the notations

$$
\begin{aligned}
& l_{\varepsilon}^{0}(\delta):=\int_{I_{\delta}^{+0}} r-\xi_{\varepsilon}^{0}-\int_{I_{\delta}^{-0}} r-\xi_{\varepsilon}^{0} \geq 0, \\
& l_{\varepsilon}^{1}(\delta):=\int_{I_{\delta}^{+1}} r-\xi_{\varepsilon}^{1}-\int_{I_{\delta}^{-1}} r-\xi_{\varepsilon}^{1} \leq 0 .
\end{aligned}
$$

and $l_{\varepsilon}(\delta)=l_{\varepsilon}^{0}(\delta)-l_{\varepsilon}^{1}(\delta) \geq 0$. The asymptotic expansions (5.12)-(5.13) follow the same procedure as in Section 4.2. The $L^{\infty}$-estimates from Section 4.3 are still valid for optima of Type II. The estimate of $\mu_{\varepsilon, \delta}$ is slightly different from the estimate in Section 4.4.

Theorem 5.1. We have the expansion:

$$
\mu_{\varepsilon, \delta}=\varepsilon \alpha^{-2}\left(h_{0}^{\prime}\left(\xi_{\varepsilon}^{0}\right) l_{\varepsilon}^{0}(\delta)+h_{0}^{\prime}\left(\xi_{\varepsilon}^{1}\right) l_{\varepsilon}^{1}(\delta)\right)+\mathcal{R}_{\mu} \text { as }(\varepsilon, \delta) \rightarrow 0,
$$

where $\mathcal{R}_{\mu}=\mathcal{O}\left(\left(\varepsilon^{1 / 2}+\delta\right) \varepsilon l_{\varepsilon}(\delta)\right)$.

Proof. We only discuss the main changes compared with the proof of Theorem 4.3. We use a Taylor expansion of $h_{0} \in \mathcal{C}^{\infty}(0,1)$ about $r=\xi_{\varepsilon}^{0}$ and $r=\xi_{\varepsilon}^{1}$ :

$$
\begin{aligned}
& h_{0}(r)=h_{0}\left(\xi_{\varepsilon}^{0}\right)+h_{0}^{\prime}\left(\xi_{\varepsilon}^{0}\right)\left(r-\xi_{\varepsilon}^{0}\right)+\frac{h_{0}^{\prime \prime}\left(\zeta^{0}\right)}{2}\left(r-\xi_{\varepsilon}^{0}\right)^{2}, \\
& h_{0}(r)=h_{0}\left(\xi_{\varepsilon}^{1}\right)+h_{0}^{\prime}\left(\xi_{\varepsilon}^{1}\right)\left(r-\xi_{\varepsilon}^{1}\right)+\frac{h_{0}^{\prime \prime}\left(\zeta^{1}\right)}{2}\left(r-\xi_{\varepsilon}^{1}\right)^{2},
\end{aligned}
$$

where $\zeta^{k} \in\left[\xi_{\varepsilon}^{k}, r\right]$ or $\zeta^{k} \in\left[r, \xi_{\varepsilon}^{k}\right]$ depending on the sign of $r-\xi_{\varepsilon}^{k}$. Plugging these Taylor expansions in $\mu_{\varepsilon, \delta}^{1}$ we consider the following expansion

$$
\mu_{\varepsilon, \delta}^{1}=\mu_{\varepsilon, \delta}^{11}+\mu_{\varepsilon, \delta}^{12}+\mu_{\varepsilon, \delta}^{13}
$$

with

$$
\begin{aligned}
\mu_{\varepsilon, \delta}^{11} & :=h_{0}\left(\xi_{\varepsilon}^{0}\right) \int_{I_{\delta}^{0}} \frac{\sigma_{\varepsilon, \delta}-\sigma_{\varepsilon, 0}}{\sigma_{\varepsilon, \delta} \sigma_{\varepsilon, 0}}+h_{0}\left(\xi_{\varepsilon}^{1}\right) \int_{I_{\delta}^{1}} \frac{\sigma_{\varepsilon, \delta}-\sigma_{\varepsilon, 0}}{\sigma_{\varepsilon, \delta} \sigma_{\varepsilon, 0}} \\
\mu_{\varepsilon, \delta}^{12} & :=h_{0}^{\prime}\left(\xi_{\varepsilon}^{0}\right) \int_{I_{\delta}^{0}} \frac{\left(\sigma_{\varepsilon, \delta}-\sigma_{\varepsilon, 0}\right)\left(r-\xi_{\varepsilon}^{0}\right)}{\sigma_{\varepsilon, \delta} \sigma_{\varepsilon, 0}}+h_{0}^{\prime}\left(\xi_{\varepsilon}^{1}\right) \int_{I_{\delta}^{1}} \frac{\left(\sigma_{\varepsilon, \delta}-\sigma_{\varepsilon, 0}\right)\left(r-\xi_{\varepsilon}^{1}\right)}{\sigma_{\varepsilon, \delta} \sigma_{\varepsilon, 0}} \\
\mu_{\varepsilon, \delta}^{13} & :=\int_{I_{\delta}^{0}} \frac{\left(\sigma_{\varepsilon, \delta}-\sigma_{\varepsilon, 0}\right) h_{0}^{\prime \prime}\left(\zeta^{0}\right)\left(r-\xi_{\varepsilon}^{0}\right)^{2}}{2 \sigma_{\varepsilon, \delta} \sigma_{\varepsilon, 0}}+\int_{I_{\delta}^{1}} \frac{\left(\sigma_{\varepsilon, \delta}-\sigma_{\varepsilon, 0}\right) h_{0}^{\prime \prime}\left(\zeta^{1}\right)\left(r-\xi_{\varepsilon}^{1}\right)^{2}}{2 \sigma_{\varepsilon, \delta} \sigma_{\varepsilon, 0}}
\end{aligned}
$$


In view of $\sigma_{\varepsilon, 0} \sigma_{\varepsilon, \delta}=\alpha(\alpha+\varepsilon)$ in $I_{\delta}^{k}$ and $\left|I_{\delta}^{+k}\right|=\left|I_{\delta}^{-k}\right|, k=0,1$ (see (5.9)) we get

$$
\int_{I_{\delta}^{k}} \frac{\sigma_{\varepsilon, \delta}-\sigma_{\varepsilon, 0}}{\sigma_{\varepsilon, \delta} \sigma_{\varepsilon, 0}}=0 \text { and } \mu_{\varepsilon, \delta}^{11}=0
$$

The computation of $\mu_{\varepsilon, \delta}^{12}$ gives

$$
\mu_{\varepsilon, \delta}^{12}=\varepsilon \alpha^{-2}\left(h_{0}^{\prime}\left(\xi_{\varepsilon}^{0}\right) l_{\varepsilon}^{0}(\delta)+h_{0}^{\prime}\left(\xi_{\varepsilon}^{1}\right) l_{\varepsilon}^{1}(\delta)\right)+\mathcal{R}_{12}(\varepsilon, \delta),
$$

with

$$
\mathcal{R}_{12}(\varepsilon, \delta)=\mathcal{O}\left(\varepsilon^{2}\left(l_{\varepsilon}^{0}(\delta)-l_{\varepsilon}^{1}(\delta)\right) .\right.
$$

All other terms in the expansion of $\mu_{\varepsilon, \delta}$ can be estimated by a straightforward adaptation of the proof of Theorem 4.3. In particular, note that thanks to (5.9) we also have $\mu_{\varepsilon, \delta}^{21}=0$ as in (4.44).

In view of (5.14) we need to show that the main term $\varepsilon \alpha^{-2}\left(h_{0}^{\prime}\left(\xi_{\varepsilon}^{0}\right) l_{\varepsilon}^{0}(\delta)+h_{0}^{\prime}\left(\xi_{\varepsilon}^{1}\right) l_{\varepsilon}^{1}(\delta)\right)$ is positive for all $\varepsilon>0$, $\delta>0$ to prove the main result. Since $\alpha>0$ and $l_{\varepsilon}^{0}(\delta) \geq 0, l_{\varepsilon}^{1}(\delta) \leq 0$ one needs to prove that $h_{0}^{\prime}\left(\xi_{\varepsilon}^{0}\right)>0$, $h_{0}^{\prime}\left(\xi_{\varepsilon}^{1}\right)<0$. The proof of Lemma 5.2 is similar to the proof of Lemma 4.4 and is left to the reader.

Lemma 5.2. If $m>\bar{m}$ then $h_{0}^{\prime}\left(\xi_{\varepsilon}^{0}\right)>0$ and $h_{0}^{\prime}\left(\xi_{\varepsilon}^{1}\right)<0$ for $\varepsilon$ small enough.

Finally we need to estimate the remainder $\mathcal{R}_{\lambda}$ as in Section 4.5. The proof follows the same ideas as in Theorem 4.5 with the difference that we need to consider the two connected components of $B_{\delta}$ here.

Theorem 5.3. We have the estimate

$$
\left|\mathcal{R}_{\lambda}\right| \leq c \varepsilon^{2} l_{\varepsilon}(\delta)\left(\left|I_{\delta}^{+}\right|^{1 / 2}+l_{\varepsilon}(\delta)\right) \text { as }(\varepsilon, \delta) \rightarrow 0,
$$

where $c>0$ is independent of $(\varepsilon, \delta)$.

Proof. Step 1 and Step 2 are identical to the corresponding steps in the proof of Theorem 5.1. Then, as in the proof of Theorem 5.1 we introduce the function

$$
F:=F_{0} F_{11}+\left(F_{11}\right)^{2}+F_{11} F_{21}=F_{11}\left(F_{0}+F_{11}+F_{21}\right) .
$$

Since the functions $u_{\varepsilon, 0}, w_{\varepsilon, \delta}, u_{\varepsilon, \delta}$ and $\mathcal{R}_{u}$ are in $\mathcal{C}^{0}(\bar{\Omega})$ due to Theorem 3.3, Lemma 4.1 and 4.2 , we get $F_{0}, F_{11}, F_{21} \in \mathcal{C}^{1}(0,1)$ and a fortiori $F \in \mathcal{C}^{1}(0,1)$. Therefore it is possible to consider the Taylor expansions

$$
\begin{aligned}
& F(r)=F\left(\xi_{\varepsilon}^{0}\right)+F^{\prime}\left(\zeta^{0}\right)\left(r-\xi_{\varepsilon}^{0}\right) \\
& F(r)=F\left(\xi_{\varepsilon}^{1}\right)+F^{\prime}\left(\zeta^{1}\right)\left(r-\xi_{\varepsilon}^{1}\right)
\end{aligned}
$$

where $\zeta^{k} \in\left[\xi_{\varepsilon}^{k}, r\right]$ or $\zeta^{k} \in\left[r, \xi_{\varepsilon}^{k}\right]$ depending on the sign of $r-\xi_{\varepsilon}^{k}, k=0,1$. This leads to

$$
\mathcal{R}_{\lambda}^{2}+\mathcal{R}_{\lambda}^{3}=\sum_{k=0,1} F\left(\xi_{\varepsilon}^{k}\right) \int_{I_{\delta}^{k}} \frac{\sigma_{\varepsilon, \delta}-\sigma_{\varepsilon, 0}}{\sigma_{\varepsilon, \delta} \sigma_{\varepsilon, 0}}+\int_{I_{\delta}^{k}} \frac{\sigma_{\varepsilon, \delta}-\sigma_{\varepsilon, 0}}{\sigma_{\varepsilon, \delta} \sigma_{\varepsilon, 0}} F^{\prime}\left(\zeta^{k}\right)\left(r-\xi_{\varepsilon}^{k}\right) .
$$

Thanks to (5.5) we have

$$
\int_{I_{\delta}^{k}} \frac{\sigma_{\varepsilon, \delta}-\sigma_{\varepsilon, 0}}{\sigma_{\varepsilon, \delta} \sigma_{\varepsilon, 0}}=0 \quad \text { for } k=0,1
$$

We also have

$$
\left|\int_{I_{\delta}^{k}} \frac{\sigma_{\varepsilon, \delta}-\sigma_{\varepsilon, 0}}{\sigma_{\varepsilon, \delta} \sigma_{\varepsilon, 0}} F^{\prime}\left(\zeta^{k}\right)\left(r-\xi_{\varepsilon}^{k}\right)\right| \leq \frac{\varepsilon l_{\varepsilon}^{k}(\delta)}{\alpha(\alpha+\varepsilon)} \sup _{r \in\left[\xi_{\varepsilon}^{k}-\delta, \xi_{\varepsilon}^{k}+\delta\right]}\left|F^{\prime}(r)\right| \leq c .
$$


The rest of the proof is similar to the corresponding part in the proof of Theorem 5.1, except that the estimates have to be written on the two components $I_{\delta}^{0}$ and $I_{\delta}^{1}$. Finally we obtain

$$
\left|\mathcal{R}_{\lambda}\right| \leq \varepsilon^{2} l_{\varepsilon}(\delta)\left(\left|I_{\delta}^{+}\right|^{1 / 2}+l_{\varepsilon}(\delta)\right)\left(c+\left|\mathcal{R}_{\lambda}\right|\right)
$$

Since $\varepsilon^{2} l_{\varepsilon}(\delta)\left(\left|I_{\delta}^{+}\right|^{1 / 2}+l_{\varepsilon}(\delta)\right)$ goes to zero when $(\varepsilon, \delta) \rightarrow 0$, we can move the term depending on $\left|\mathcal{R}_{\lambda}\right|$ in the right-hand side of (5.22) to the left-hand side and we obtain (5.20).

Finally we obtain the main result for optimizers of Type II.

Theorem 5.4. If $m>\bar{m}$ there exists $\varepsilon_{0}>0$ such that for all $B \in \mathcal{B}$ and for all $0<\varepsilon<\varepsilon_{0}$ there exists $\xi_{\varepsilon}^{0}$, $\xi_{\varepsilon}^{1}$ such that

$$
\lambda_{\varepsilon}\left(B_{\varepsilon}^{*}\right) \leq \lambda_{\varepsilon}(B)
$$

where $B_{\varepsilon}^{*}=B_{\varepsilon, 1}^{*} \cup B_{\varepsilon, 2}^{*}$ with

$$
\begin{aligned}
& B_{\varepsilon, 1}^{*}=\mathrm{B}\left(0, \xi_{\varepsilon}^{0}\right), \\
& B_{\varepsilon, 2}^{*}=\mathrm{B}(0,1) \backslash \overline{\mathrm{B}\left(0, \xi_{\varepsilon}^{1}\right)},
\end{aligned}
$$

and the equality occurs only when $B=B_{\varepsilon}^{*}$ almost everywhere in $\Omega$. In addition we have

$$
\left(\xi_{\varepsilon}^{0}, \xi_{\varepsilon}^{1}\right) \rightarrow\left(\xi^{0}, \xi^{1}\right) \text { as } \varepsilon \rightarrow 0 .
$$

Proof. The proof is similar to the proof of Theorem 4.6 and is left to the reader. The convergence (5.25) is readily seen from the fact that $\delta(\varepsilon) \rightarrow 0$ as $\varepsilon \rightarrow 0$ and from (5.6) we get $\left|\xi_{\varepsilon}^{k}-\xi^{k}\right| \leq \delta(\varepsilon)$ for $k=0,1$.

\section{Conclusion}

In this paper we have exhibited global minimizers of the ground state for two phase conductors in low contrast regime, i.e. when $\beta-\alpha=\varepsilon$ is small, and when $\Omega=\mathbb{B}(0,1)$. The result have been obtained in two and three dimensions for simplicity but can be easily extended to higher dimensions, the only difference residing in the solutions of the one-dimensional equation (3.1)-(3.2). The global minimum depends on the volume constraint $|B|=m$. When $m<\bar{m}$ the minimizer is a centered disk, and when $m>\bar{m}$ the minimizer is the union of a centered disk and of a centered ring touching the boundary of $\Omega$. When $m>\bar{m}$, the global optimizer depends on $\varepsilon$, which was to be expected since the optimal set has two connected components. In the general case, i.e. when $\beta>0$ and $\alpha>0$ are arbitrary, we conjecture that the global minimizers are also of this nature, i.e. of Type I or of Type II as introduced in Proposition 3.1, and that the threshold $\bar{m}$ depends on $(\alpha, \beta)$. However, the strategy developed in this paper cannot be applied in the general case and a new idea should be found to solve this question.

It does not seem that the technique used in this paper can be extended to the case where $\Omega$ is not a ball. Indeed the foundation of our result is the convergence of the gradient in $L^{\infty}(\Omega)$ in Theorem 3.3 which relies on the additional regularity provided by the radial symmetry of the problem. Without this symmetry, the convergence is unlikely as the gradient may have singularities for a nonsmooth set $B$. Even if the eigenvalue can be lowered as in Theorems 3.7 and 3.9, it is not even known if problem (1.4)-(1.5) has a solution so we could not prove yet a result such as Corollary 3.10. Consequently for a general $\Omega$ the primary mathematical question is to determine the existence of a solution to (1.4)-(1.5).

\section{REFERENCES}

[1] A. Alvino, G. Trombetti and P.-L. Lions, On optimization problems with prescribed rearrangements. Nonlinear Anal. 13 (1989) $185-220$.

[2] P.R. Beesack, Hardy's inequality and its extensions. Pacific J. Math. 11 (1961) 39-61. 
[3] C. Conca, A. Laurain and R. Mahadevan, Minimization of the ground state for two phase conductors in low contrast regime. SIAM J. Appl. Math. 72 (2012) 1238-1259.

[4] C. Conca, R. Mahadevan and L. Sanz, An extremal eigenvalue problem for a two-phase conductor in a ball. Appl. Math. Optim. 60 (2009) 173-184.

[5] C. Conca, R. Mahadevan and L. Sanz, Shape derivative for a two-phase eigenvalue problem and optimal configurations in a ball, in vol. 27 of CANUM 2008, ESAIM Proc. EDP Sciences, Les Ulis (2009) 311-321

[6] S. Cox and R. Lipton, Extremal eigenvalue problems for two-phase conductors. Arch. Rational Mech. Anal. 136 (1996) $101-117$.

[7] M. Dambrine and D. Kateb, On the shape sensitivity of the first Dirichlet eigenvalue for two-phase problems. Appl. Math. Optim. 63 (2011) 45-74.

[8] G.H. Hardy, J.E. Littlewood and G. Pólya, Inequalities, Cambridge Mathematical Library. Cambridge University Press, Cambridge (1988). Reprint of the 1952 edition.

[9] A. Henrot, Extremum problems for eigenvalues of elliptic operators, Frontiers in Mathematics. Birkhäuser Verlag, Basel (2006).

[10] M.G. Krein, On certain problems on the maximum and minimum of characteristic values and on the Lyapunov zones of stability. Amer. Math. Soc. Transl. 1 (1955) 163-187.

[11] M.G. Krein and M.A. Rutman, Linear operators leaving invariant a cone in a banach space. Amer. Math. Soc. Transl. (1950) 26.

[12] F Rellich, Perturbation Theory of Eigenvalue Problems, Notes on mathematics and its applications. Gordon and Breach, New York (1969).

[13] G.N. Watson, A Treatise on the Theory of Bessel Functions. Cambridge University Press, Cambridge, England (1944). 